\title{
Oxidative Coupling of Aryl Boronic Acids with Aryl- and Alkylamines via Cooperative Photoredox and Copper Catalysis.
}

\author{
Jennifer R. Marshall, Juliana N. O’Brien, and Andrew J. Wommack ${ }^{*}$
}

Department of Chemistry, High Point University, High Point, NC 27268

\section{Corresponding Author}

E-mail: awommack@highpoint.edu

Table of Contents/Abstract Graphic

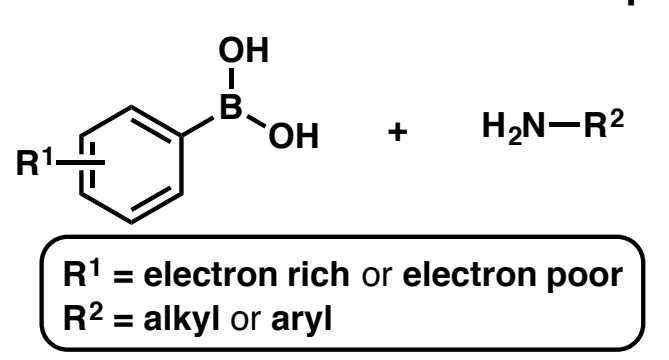

$\mathrm{Cu}(\mathrm{acac})_{2}(10 \mathrm{~mol} \%)$

$\mathrm{Ru}(\mathrm{bpy})_{3}\left(\mathrm{PF}_{6}\right)_{2}(1.0 \mathrm{~mol} \%)$

2,6-lutidine

$\underset{452 \mathrm{~nm}, \text { Blue LEDs }}{\stackrel{\text { toluene/acetonitrile }}{\longrightarrow}}$

- safe, mild conditions

ambient atm, $20 \mathrm{~h}, 35^{\circ} \mathrm{C} \quad$ - moderate to excellent yields

\section{Abstract}

Copper(II)-catalyzed oxidative cross-couplings of aryl boronic acids with aryl- and alkylamines have been accomplished across a range of substrates in the presence of a ruthenium(II)-photoredox cocatalyst under mild aerobic conditions. This modified $\mathrm{C}-\mathrm{N}$ cross-coupling reaction allows the incorporation of alkylamines to both electron poorand electron-rich aryl boronic acids with low photocatalyst loadings and under ambient atmosphere. The coupling protocol provides secondary amines in yields of $63-90 \%$ during the safe, procedurally improved process. 
The pervasive occurrence of the carbon-nitrogen bond in organic molecules has inspired many chemists to develop efficient, economical methods for producing these highly important bonds. ${ }^{1}$ Although widely regarded as powerful $\mathrm{C}-\mathrm{N}$ coupling methods, palladium- or nickel-catalyzed coupling reactions of amines with aryl halides typically require rigorously inert atmosphere conditions, heating, and oftentimes involve expensive metal catalysts. ${ }^{2}$ The vast majority of chemists have embraced these operational costs due to low catalyst loadings and generally tolerating quite a range of functional group diversity on the coupling partners. Efforts to improve these costs with copper-mediated $\mathrm{C}-\mathrm{N}$ coupling protocols have developed at a comparatively slower progression than the well-known Pd-based methods. ${ }^{3}$ However, recent developments in both copper and photoredox catalysis inspired our interest in the further development of catalytic $\mathrm{C}-\mathrm{N}$ forming strategies. ${ }^{4}$

Shown in Figure $1 \mathrm{~A}$ are examples of pharmaceutically relevant molecules that can utilize $\mathrm{Pd}$-catalyzed $\mathrm{C}-\mathrm{N}$ coupling reactions, which requires subsequent rounds of purification to remove $\mathrm{Pd}$ and thereby adding undesirable expense. ${ }^{5}$ Efforts to further develop the copper-catalyzed Chan-Lam reaction have typically involved incorporation of additives, oxygen saturation of the reaction mixture, and heating (Figure 1B). ${ }^{6}$ Inspired by the work of Kobayashi and coworkers, who enlisted photochemical properties of $\mathrm{fac}-\left[\operatorname{lr}(\mathrm{ppy})_{3}\right]$ to couple aniline derivatives with aryl boronic acids, we sought to further improve this protocol by expanding the substrate scope to include alkyl amines while exploring alternative photoredox catalysts and copper(II) sources. 


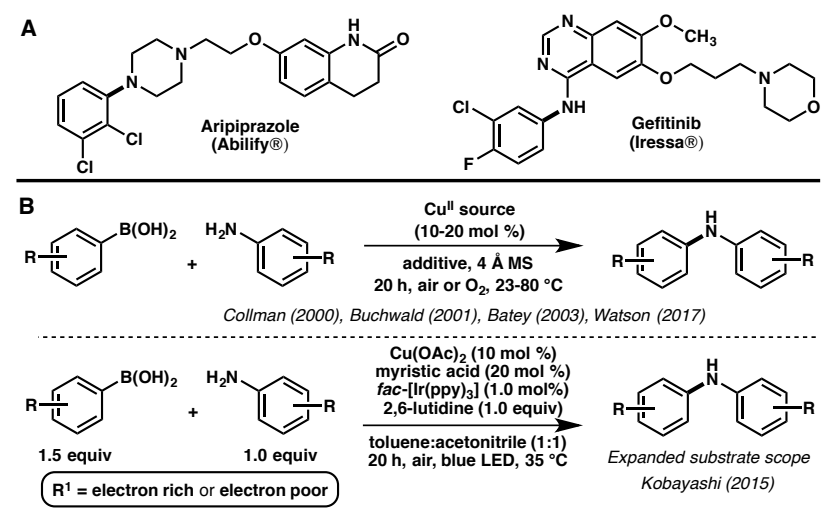

Figure 1. A) Synthetic targets with $\mathrm{C}-\mathrm{N}$ coupling sites shown in bold. B) Prior advances in Cu"-catalyzed reaction methods.

Our initial trials centered around reproducing the blended Kobayashi-Buchwald methodology while varying the photoredox catalyst or copper(II) source. While many other photocatalysts were also investigated, shown in Table 1 is a select set of our trials that describes comparable efficiencies between fac-[Ir(ppy $\left.)_{3}\right], \quad R u(b p z)_{3}\left(\mathrm{PF}_{6}\right)_{2}$, $\mathrm{Ru}(\mathrm{bpy})_{3} \mathrm{Cl}_{2}$, and $\mathrm{Ru}(\mathrm{bpy})_{3}\left(\mathrm{PF}_{6}\right)_{2}$ (See Figures $\mathrm{S} 1$, S2, Tables S1, S2, S3 in Supporting Information). The $\left[\mathrm{Ru}(\mathrm{bpy})_{3}\right]^{2+}$ source exhibited quite different solubilities depending on the counterion, with the hexafluorophosphate catalyst being much more soluble. The observed difference in reaction outcome is attributed to this property (Table 1, entries 5 \& 6). With the soluble $\left[\mathrm{Ru}(\mathrm{bpy})_{3}\right]^{2+}$ photocatalyst proving to be productive in this screen (Table 1, entry 6), copper(II) sources were investigated to retain the solubility and reactivity profile of the $\mathrm{Cu}(\mathrm{OAc})_{2}$ and myristic acid combination. It was found that $\mathrm{Cu}(\mathrm{acac})_{2}$ performed at a comparable level, but further steric hindrance around the hydrocarbon-based ligand did not produce the same reaction efficiency. Irradiating the photocatalyst in the reaction mixture in the absences of $\mathrm{Cu}(\mathrm{acac})_{2}$ proved to make the 
phenol from the arylboronic acid progenitor, as previously disclosed by Jørgensen, Xiao, and coworkers. ${ }^{7}$ Attempts to enhance reaction performance by including fluorinated ligand derivatives on copper(II) did not prove beneficial.

Table 1. Select Examples of Alternative Copper(II) Sources and Photocatalysts

\begin{tabular}{|c|c|c|c|c|}
\hline \multirow[b]{2}{*}{ entry } & \multirow[b]{2}{*}{ copper source } & \multicolumn{2}{|c|}{$\begin{array}{c}\text { Cull source } \\
\text { photocatalyst } \\
2,6-\text {-lutidine } \\
\text { toluene/acetonitrile } \\
\text { Blue LED } \\
20 \mathrm{~h} \text {, open air }\end{array}$} & \multirow[b]{2}{*}{ yield } \\
\hline & & photocatalyst & additive & \\
\hline $1^{b}$ & $\begin{array}{l}\mathrm{Cu}(\mathrm{OAc})_{2} \\
(10 \mathrm{~mol} \%)\end{array}$ & none & none & 8 \\
\hline 2 & $\begin{array}{l}\mathrm{Cu}(\mathrm{OAc})_{2} \\
(10 \mathrm{~mol} \%)\end{array}$ & none & $\begin{array}{c}\text { myristic acid } \\
(20 \mathrm{~mol} \%)\end{array}$ & 27 \\
\hline 3 & $\begin{array}{l}\mathrm{Cu}(\mathrm{OAC})_{2} \\
(10 \mathrm{~mol} \%)\end{array}$ & $f a c-\left[\mathrm{Ir}(\mathrm{ppy})_{3}\right]$ & $\begin{array}{c}\text { myristic acid } \\
(20 \mathrm{~mol} \%)\end{array}$ & 92 \\
\hline 4 & $\begin{array}{l}\mathrm{Cu}(\mathrm{OAc})_{2} \\
(10 \mathrm{~mol} \%)\end{array}$ & $\begin{array}{c}\mathrm{Ru}(\mathrm{bpz})_{3}\left(\mathrm{PF}_{6}\right)_{2} \\
(1 \mathrm{~mol} \%)\end{array}$ & $\begin{array}{c}\text { myristic acid } \\
(20 \mathrm{~mol} \%)\end{array}$ & 90 \\
\hline 5 & $\begin{array}{l}\mathrm{Cu}(\mathrm{OAc})_{2} \\
(10 \mathrm{~mol} \%)\end{array}$ & $\underset{(1 \mathrm{~mol} \%)}{\mathrm{Ru}(\mathrm{bpy})_{3} \mathrm{Cl}_{2}}$ & $\begin{array}{c}\text { myristic acid } \\
(20 \mathrm{~mol} \%)\end{array}$ & 79 \\
\hline 6 & $\begin{array}{l}\mathrm{Cu}(\mathrm{OAc})_{2} \\
(10 \mathrm{~mol} \%)\end{array}$ & $\begin{array}{c}\mathrm{Ru}(\mathrm{bpy})_{3}\left(\mathrm{PF}_{6}\right)_{2} \\
(1 \mathrm{~mol} \%)\end{array}$ & $\begin{array}{c}\text { myristic acid } \\
(20 \mathrm{~mol} \%)\end{array}$ & 91 \\
\hline $7^{b}$ & $\begin{array}{l}\mathrm{Cu}(\mathrm{OAC})_{2} \\
(10 \mathrm{~mol} \%)\end{array}$ & $\begin{array}{c}\mathrm{Ru}(\mathrm{bpy})_{3}\left(\mathrm{PF}_{6}\right)_{2} \\
(1 \mathrm{~mol} \%)\end{array}$ & $\underset{(20 \mathrm{~mol} \%)}{\text { myristic acid }}$ & 15 \\
\hline 8 & $\begin{array}{l}\mathrm{Cu}(\mathrm{acac})_{2} \\
(10 \mathrm{~mol} \%)\end{array}$ & $\begin{array}{c}\mathrm{Ru}(\mathrm{bpy})_{3}\left(\mathrm{PF}_{6}\right)_{2} \\
(1 \mathrm{~mol} \%)\end{array}$ & none & 89 \\
\hline $9^{b}$ & $\begin{array}{l}\mathrm{Cu}(\mathrm{acac})_{2} \\
(10 \mathrm{~mol} \%)\end{array}$ & $\begin{array}{c}\mathrm{Ru}(\mathrm{bpy})_{3}\left(\mathrm{PF}_{6}\right)_{2} \\
(1 \mathrm{~mol} \%)\end{array}$ & none & 14 \\
\hline $10^{c}$ & none & $\begin{array}{c}\mathrm{Ru}(\mathrm{bpy})_{3}\left(\mathrm{PF}_{6}\right)_{2} \\
(1 \mathrm{~mol} \%)\end{array}$ & none & N.D. \\
\hline 11 & $\begin{array}{l}\mathrm{Cu}(\mathrm{TMHD})_{2} \\
(10 \mathrm{~mol} \%)^{2}\end{array}$ & $\begin{array}{c}\mathrm{Ru}(\mathrm{bpy})_{3}\left(\mathrm{PPF}_{6}\right)_{2} \\
(1 \mathrm{~mol} \%)\end{array}$ & none & 54 \\
\hline 12 & $\frac{\mathrm{Cu}(2 \text {-ethylhexanoate) }}{(10 \mathrm{~mol} \%)}$ & $\begin{array}{c}\mathrm{Ru}(\mathrm{bpy})_{3}\left(\mathrm{PF}_{6}\right)_{2} \\
(1 \mathrm{~mol} \%)\end{array}$ & none & 47 \\
\hline 13 & $\begin{array}{l}\mathrm{Cu}(\mathrm{FOD})_{2} \\
(10 \mathrm{~mol} \%)\end{array}$ & $\begin{array}{c}\mathrm{Ru}(\mathrm{bpy})_{3}\left(\mathrm{PF}_{6}\right)_{2} \\
(1 \mathrm{~mol} \%)\end{array}$ & none & 24 \\
\hline 14 & $\begin{array}{l}\text { Cu(hfacac })_{2} \\
(10 \mathrm{~mol} \%)\end{array}$ & $\begin{array}{c}\mathrm{Ru}(\mathrm{bpy})_{3}\left(\mathrm{PF}_{6}\right)_{2} \\
(1 \mathrm{~mol} \%)\end{array}$ & none & 31 \\
\hline
\end{tabular}

${ }^{a}$ Isolated yields from chromatography using 4-chlorophenylboronic acid (1.50 equiv), aniline (1.00 equiv), $\mathrm{Cu}^{\prime \prime}$ source $(10 \mathrm{~mol} \%)$, photocatalyst $(1.0 \mathrm{~mol} \%)$, 2,6-lutidine (2.00 equiv), toluene/MeCN (1:1, v/v), $35{ }^{\circ} \mathrm{C}, 20 \mathrm{~h}$, ambient air, blue LED irradiation. ${ }^{b}$ Reactions were heated at $35{ }^{\circ} \mathrm{C}$ and not irradiated. ${ }^{c}$ Complete consumption of the 4-chlorophenylboronic acid. N.D. = not detected.

Using the simplified reaction conditions shown in entry 8 of Table 1, our tests of reaction scope began with coupling a diverse array of aryl amines with 4chlorophenylboronic acid (Table 2), which was shown to be a problematic coupling partner in previous studies, presumably due to slow transmetalation from the electronpoor arylboronic acid. ${ }^{6 c}$ Entries 1-8 display the dependence of the photocatalyst in the 
reaction outcome when varying electronic properties of the aniline derivative, where the electron-poor derivatives are dramatically impacted by the presence of the co-catalyst. Additionally, the yield for 7 proved reproducible when tested on a larger scale $(0.936 \mathrm{~g}$ of the boronic acid). Increasing steric hindrance around the nitrogen coupling partner and chemoselectivity were also probed in entries 9 and 10, respectively.

Table 2. Coupling of 4-Chlorophenylboronic Acid with Arylamines

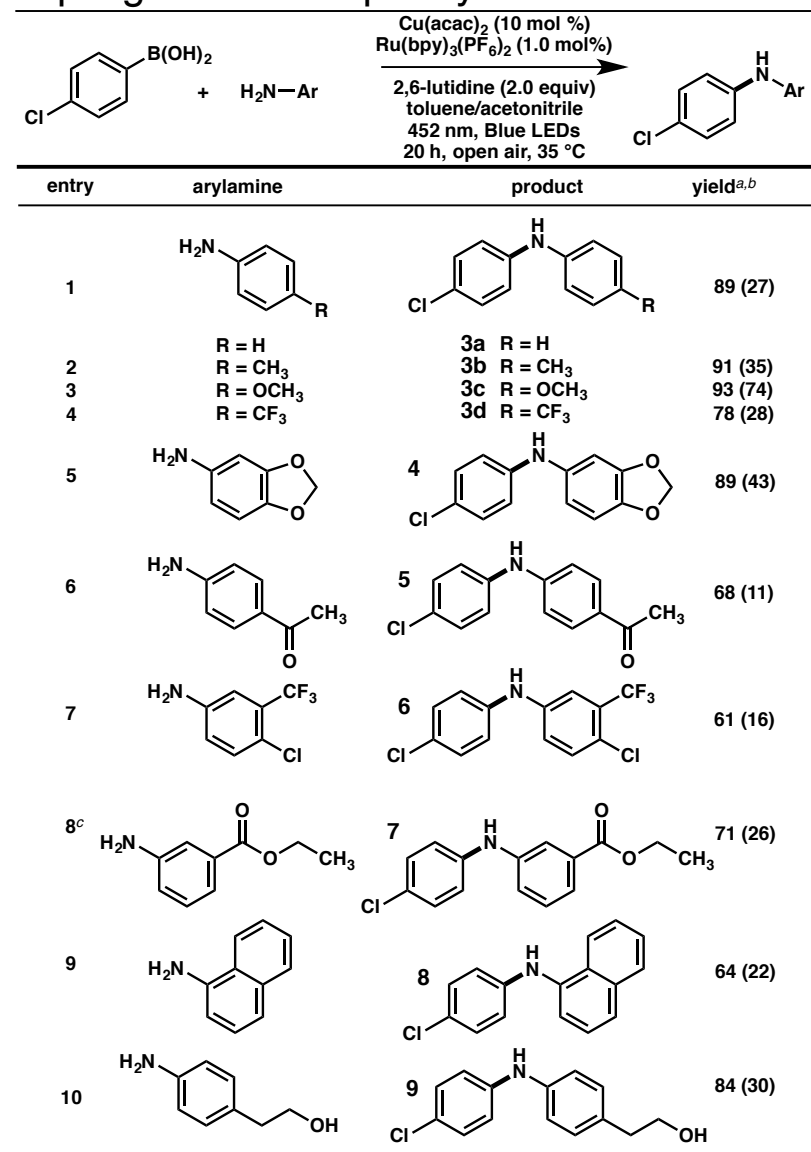

${ }^{a}$ The reaction was performed with boronic acid (1.50 equiv), amine (1.00 equiv), $\mathrm{Cu}(\mathrm{acac})_{2}(10 \mathrm{~mol} \%)$, $\mathrm{Ru}(\mathrm{bpy})_{3}\left(\mathrm{PF}_{6}\right)_{2}(1.0 \mathrm{~mol} \%), 2,6$-lutidine $\left(2.0\right.$ equiv), toluene/MeCN $(1: 1, \mathrm{v} / \mathrm{v}), 35^{\circ} \mathrm{C}, 20 \mathrm{~h}$, ambient air, blue LED irradiation. ${ }^{b}$ Yields are shown in the following order: yield with $\mathrm{Ru}(\mathrm{bpy})_{3}\left(\mathrm{PF}_{6}\right)_{2}$ and, in parentheses, without photocatalyst. All reactions are with continuous blue LED irradiation. Percent yields are isolated yields when using photocatalyst, and for reactions without photocatalyst, yields are derived from LC-MS, using 4-bromodiphenylamine as the internal standard. ${ }^{C}$ Reaction was also run at larger scale. See Experimental Section for details. 
To test the tolerance of the arylboronic acid coupling partner, either aniline or 4chloroaniline was used, as shown in Table 3. Steric hindrance added to the arylboronic acid does affect the reaction efficiency, as seen in entry 5 . However, product $\mathbf{1 4}$ is still isolated in moderate yield. Upon comparison of this result to the reaction trial in the absence of photocatalyst, a $63 \%$ isolated yield is a dramatic improvement. The pyridine derivative is formed in a $73 \%$ yield with these conditions, but despite our repeated attempts, no commercially available thiophene-based boronic acid underwent smooth conversion to the desired secondary aromatic amine product.

Table 3. Coupling of Arylamines with Arylboronic Acids

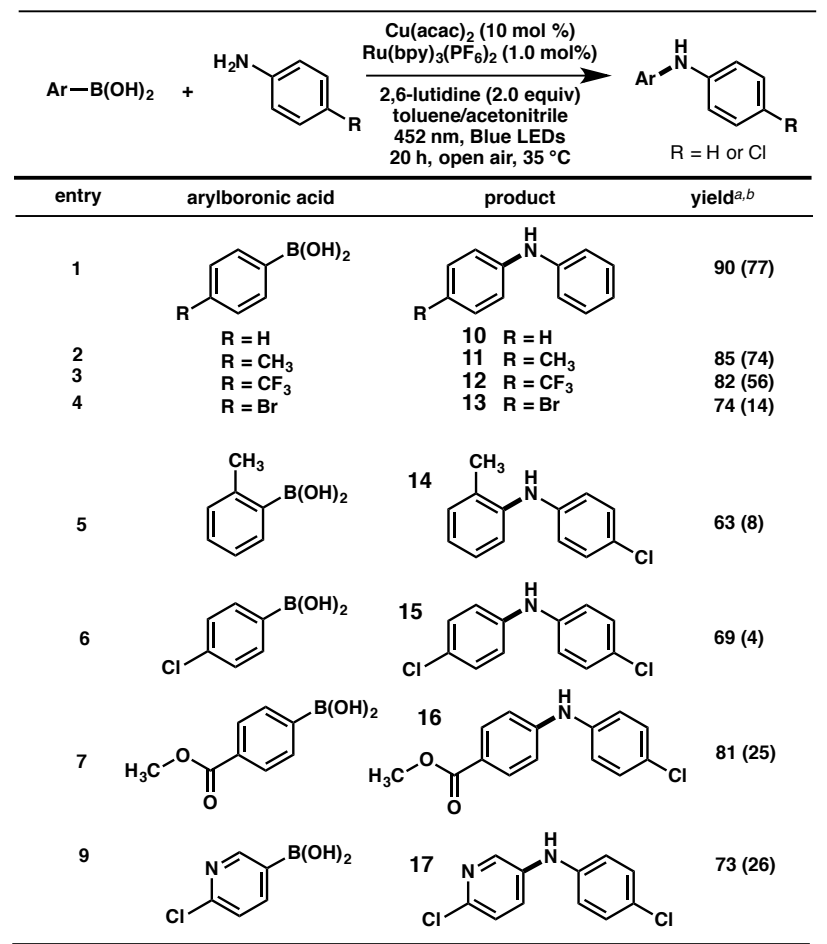

${ }^{a}$ The reaction was performed with boronic acid (1.50 equiv), amine (1.00 equiv), $\mathrm{Cu}(\mathrm{acac})_{2}(10 \mathrm{~mol} \%)$, $\mathrm{Ru}(\mathrm{bpy})_{3}\left(\mathrm{PF}_{6}\right)_{2}(1.0 \mathrm{~mol} \%), 2,6$-lutidine (2.0 equiv), toluene/MeCN $(1: 1, \mathrm{v} / \mathrm{v}), 35^{\circ} \mathrm{C}, 20 \mathrm{~h}$, ambient air, blue LED irradiation. ${ }^{b}$ Yields are shown in the following order: yield with $\mathrm{Ru}(\mathrm{bpy})_{3}\left(\mathrm{PF}_{6}\right)_{2}$ and, in parentheses, without photocatalyst. All reactions are with continuous blue LED irradiation. Percent yields are isolated yields when using photocatalyst, and for reactions without photocatalyst, yields are derived from LC-MS, using 4-bromodiphenylamine as the internal standard. 
Although prior studies have shown alkylamine substrates can undergo productive union with arylboronic acids, typically these reactions require electron-rich or neutral arylboronic acids, alternative reaction additives, fully oxygenated atmospheres, and additional heating. ${ }^{6}$ Table 4 demonstrates that in the presence of the soluble copper(II) catalyst, the efficiency of the desired $\mathrm{C}-\mathrm{N}$ union is greatly impacted by the photocatalyst. Entries 2-4 show effective merger of branched primary amines, entries 5 and 6 show alkene-containing alkylamines are tolerated, and increasing steric hindrance with the secondary amine piperidine and 2-methylpiperidine is incorporated to form tertiary amine products. Although the products $\mathbf{2 4}$ and $\mathbf{2 5}$ are obtained in $81 \%$ and $67 \%$ yields, respectively, the presence of tertiary amine reaction products from continued reaction in entries 1-6 and 9-11 was not observed. Entry 3 was also completed on a larger scale, which resulted in a similar production of 20. Oxygen functionality in the form of ether or ester is well tolerated, as shown in entries 9-11; however, attempts to use carboxylic acids in aliphatic amino acids proved unfavorable. This shortcoming may be due to the known decarboxylation that can occur in the presence of a sufficiently oxidizing photocatalyst. $^{8}$

Table 4. Coupling of 4-Chlorophenylboronic Acid with Alkylamines 


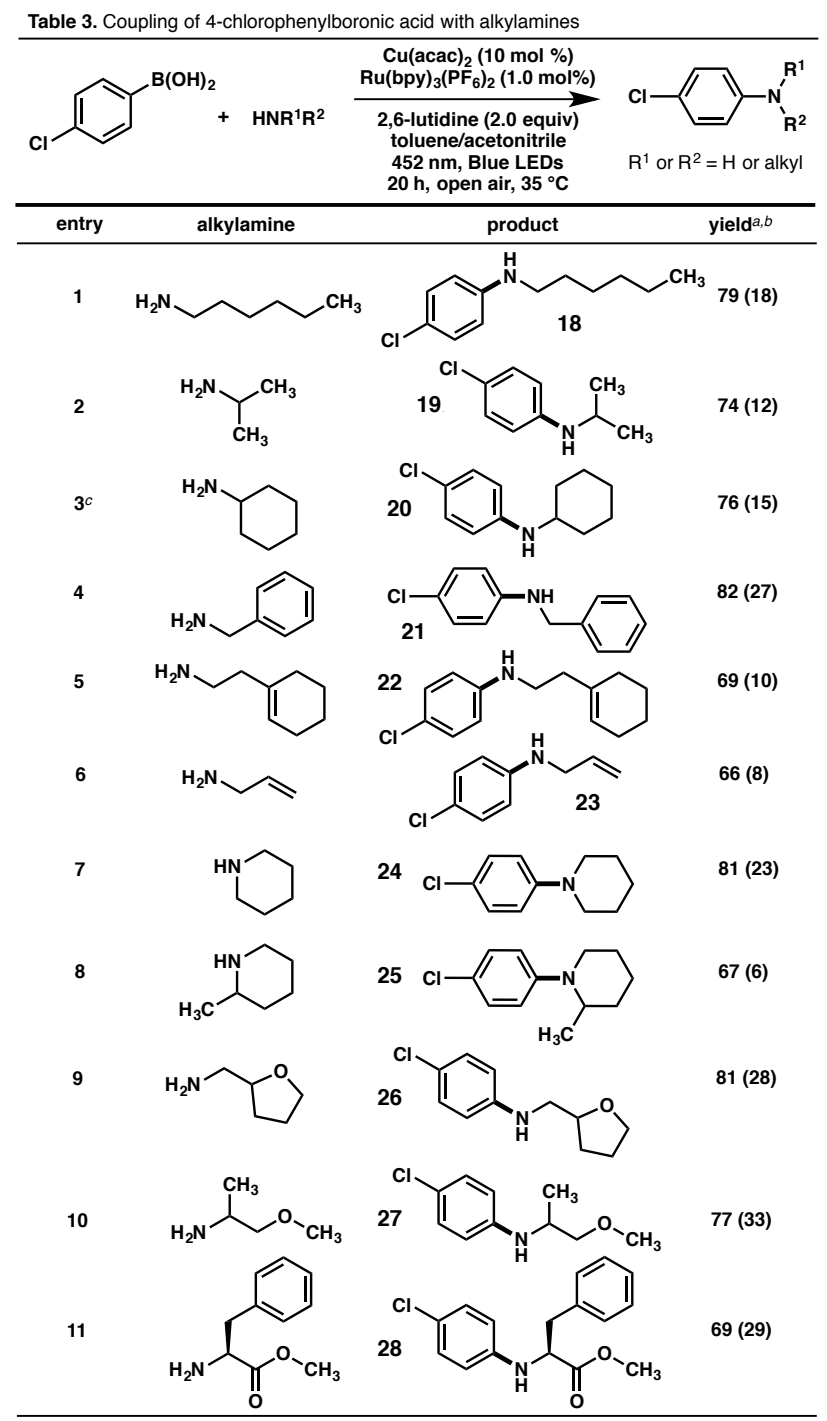

${ }^{a}$ The reaction was performed with boronic acid (1.50 equiv), amine (1.00 equiv), $\mathrm{Cu}(\mathrm{acac})_{2}(10 \mathrm{~mol} \%)$, $\mathrm{Ru}(\mathrm{bpy})_{3}\left(\mathrm{PF}_{6}\right)_{2}(1.0 \mathrm{~mol} \%), 2,6$-lutidine (2.0 equiv), toluene/MeCN $(1: 1, \mathrm{v} / \mathrm{v}), 20 \mathrm{~h}$, ambient air, blue LED irradiation, $35^{\circ} \mathrm{C}$. ${ }^{b}$ Yields are shown in the following order: yield with $\mathrm{Ru}(\mathrm{bpy})_{3}\left(\mathrm{PF}_{6}\right)_{2}$ and, in parentheses, without photocatalyst. All reactions are with continuous blue LED irradiation. Percent yields are isolated yields when using photocatalyst, and for reactions without photocatalyst, yields are derived from LC-MS, using 4-bromodiphenylamine as the internal standard. ${ }^{c}$ Reaction was also run at larger scale. See Experimental Section for details.

Studies into the mechanistic process for the coupling of boronic esters with amines by Watson and coworkers built upon earlier reports by the Evans and Stahl Labs, during their studies of the copper(II)-promoted etherification reaction with boronic acids. ${ }^{6 g, 9}$ Found in these literature examples are important aspects to help suppress byproduct formation in the current reaction protocol involving boronic acids, such as limiting 
excess water, increasing reactivity through amine assisted decomplexation, and facilitating rapid re-oxidation of copper following reductive elimination of desired product. We found that a critical and operationally simple step in our reaction assembly was to use solvent that had been stored over activated $4 \AA$ molecular sieves. The reduction in water content was important to reduce the undesired, copper-mediated conversion of the boronic acid reagents to the corresponding phenolic compounds. ${ }^{6 g, 9 a}$ Using 2.0 equivalents of the toluidine base produced reliable reaction yields by facilitating improved aryl- and alkylamine complexation to copper(II). Finally, Watson and coworkers demonstrated the need for enhanced re-oxidation of copper(I) after reductive elimination of the newly formed $\mathrm{C}-\mathrm{N}$ union. Extended exposure of boronic esters to copper(I) increased protodeboronation and, if water is present in appreciable amounts, oxidative deboronation and ether formation can result. ${ }^{6 g}$ It is with these side products in mind that we undertook Stern-Volmer analyses of copper(I) acetate, as a surrogate for the copper(I) species formed with $\mathrm{Cu}(\mathrm{acac})_{2}$ during the putative catalytic cycle (See Figure $\mathrm{S} 6$ in Supporting Information). Using argon-degassed $\mathrm{Ru}(\mathrm{bpy})_{3} \mathrm{Cl}_{2}$ in toluene:MeCN $(1: 1, \quad v / v), \mathrm{Cu}(\mathrm{OAc})$ does efficiently quench the photocatalyst fluorescence, which suggests a means for improving copper(I) re-oxidation in our reaction conditions and also suggesting a reason for the low observed levels of protodeboronation. In prior trifluoromethylation studies by the Sanford and MacMillan Labs, the productive interaction of the excited state of $\left[\mathrm{Ru}(\mathrm{bpy})_{3}\right]^{2+}$ with $\mathrm{Cu}^{\prime} \rightarrow \mathrm{Cu}^{\prime \prime}$ is proposed, as this interaction is reported to be thermodynamically favorable..$^{10,11}$ 
Additional routes to facilitate copper $(\mathrm{I})$ oxidation can involve singlet oxygen $\left({ }^{1} \mathrm{O}_{2}\right)$, which is known to be produced during photocatalysis. ${ }^{12}$ To test if singlet oxygen was significantly involved in the $\mathrm{Cu}^{\prime} \rightarrow \mathrm{Cu}^{\prime \prime}$ oxidation event, we conducted a series of model reactions using our standard conditions in the presence of known ${ }^{1} \mathrm{O}_{2}$ traps (See Table S3 in Supporting Information). Reaction efficiencies were slightly reduced, which does not completely rule out ${ }^{1} \mathrm{O}_{2}$ involvement in copper redox events during the reaction. However, these results combined with the fluorescence quenching studies suggest that the copper(I) oxidation is being affected by the photooxidative capacity of the $\left[\mathrm{Ru}(\mathrm{bpy})_{3}\right]^{2+}$ catalyst. Further complications from ambient levels of molecular oxygen include superoxide formation during $\left[\mathrm{Ru}(\mathrm{bpy})_{3}\right]^{2+}$ irradiation, which does produce oxidative hydroxylation products from the corresponding boronic acid reactants in the absence of the copper(II) catalyst (Table 1, entry 8 ). However, we do not observe appreciable formation of phenol byproducts under standard reaction conditions.

In conclusion, a modified procedure for the copper-catalyzed Chan-Lam reaction of arylamines or alkylamines with arylboronic acids was developed. Through the productive merger of copper and photoredox catalysis, the substrate scope of this oxidative coupling reaction was expanded to include electron-deficient aryl boronic acids as viable starting materials under ambient atmosphere.

\section{Experimental Section}

General Information: All reagents were purchased and used as received. Solvents were stored over activated $4 \AA$ AS. LED lights were purchased from Creative Lighting 
Solutions (www.creativelightings.com) and assembled to produce a photon flux density of $460 \mu \mathrm{mol} \cdot \mathrm{m}^{-2} \cdot \mathrm{sec}^{-1}$ (see Figure S1 in Supporting Information). Reaction vessels were placed against the LED assembly, which provided minor heating to elevate the reaction temperature to $35{ }^{\circ} \mathrm{C}$. $\quad{ }^{1} \mathrm{H}$ and ${ }^{13} \mathrm{C}$ shifts are reported in ppm with the solvent resonance as the internal standard. ${ }^{1} \mathrm{H}$ NMR data are reported as follows: chemical shift, multiplicity $(\mathrm{s}=$ singlet, $\mathrm{d}=$ doublet, $\mathrm{t}=$ triplet, $\mathrm{q}=$ quartet, $\mathrm{br}=$ broad, $\mathrm{m}=$ multiplet), coupling constants $(\mathrm{Hz})$, and integration. Infrared spectra were recorded on an FT-IR spectrophotometer as thin films using ATR attachment; peaks are reported in units of wavenumbers $\left(\mathrm{cm}^{-1}\right)$ as strong (s), medium (m), weak (w), and broad (br). Highresolution mass spectra (HRMS) were obtained using ESI-TOF.

\section{General Procedure for the Coupling of Amines with Aryl boronic Acids by Cooperative Photoredox and Copper Catalysis.}

4-Chloro- $N$-phenylaniline $(\mathbf{3 a}) \cdot{ }^{13}$ In a 1.5 dram screw-cap vial was added $\mathrm{Ru}(\mathrm{bpy})_{3}\left(\mathrm{PF}_{6}\right)_{2}$ $(0.0086 \mathrm{~g}, 0.010 \mathrm{mmol}, 1.0 \mathrm{~mol} \%), \mathrm{Cu}(\mathrm{acac})_{2}(0.0262 \mathrm{~g}, 0.100 \mathrm{mmol}, 10 \mathrm{~mol} \%), 4-$ chlorophenylboronic acid $(0.234 \mathrm{~g}, 1.50 \mathrm{mmol}, 1.50$ equiv), aniline $(0.0913 \mathrm{~mL}, 1.00 \mathrm{mmol}$, 1.00 equiv), 2,6-lutidine ( $0.231 \mathrm{~mL}, 2.00 \mathrm{mmol}, 2.0$ equiv), and toluene:MeCN (1:1, $3.00 \mathrm{~mL})$. The vial was closed with a teflon-lined cap and was stirred for $20 \mathrm{~h}$ under blue LED irradiation. (Heat generated by the LED lamp elevated the temperature to $35^{\circ} \mathrm{C}$.) Following the reaction period, the crude reaction mixture was passed through a plug of silica gel with 1:1 hexanes/ethyl acetate rinses, concentrated under reduced pressure, and further purified with silica gel flash chromatography (hexanes/ethyl acetate, 20:1, $R_{f}=0.35$ ) to deliver $3 a$ as a white solid $(0.181 \mathrm{~g}, 0.890 \mathrm{mmol}, 89 \%) .{ }^{1} \mathrm{H}$ NMR $\left(400 \mathrm{MHz}, \mathrm{CDCl}_{3}\right): \delta 7.32-7.22(\mathrm{~m}, 2 \mathrm{H}), 7.22-$ 
$7.15(\mathrm{~m}, 2 \mathrm{H}), 7.04-7.01(\mathrm{~m}, 2 \mathrm{H}), 7.00-6.90(\mathrm{~m}, 3 \mathrm{H}), 5.64(\mathrm{~s}, 1 \mathrm{H}) \cdot{ }^{13} \mathrm{C}$ NMR $(100 \mathrm{MHz}$, $\left.\mathrm{CDCl}_{3}\right): \delta 142.77,141.99,129.57,129.39,125.61,121.64,118.93,118.23$. HRMS (ESI+) Calcd for $\mathrm{C}_{12} \mathrm{H}_{11} \mathrm{CIN}^{+}[\mathrm{M}+\mathrm{H}]^{+}$: 204.0575; Found 204.0561.

4-Chloro- $N$-( $p$-tolyl)aniline $\quad(\mathbf{3 b}) .{ }^{14}$ The general procedure was followed using $\mathrm{Ru}(\mathrm{bpy})_{3}\left(\mathrm{PF}_{6}\right)_{2}(0.0086 \mathrm{~g}, 0.010 \mathrm{mmol}, 1.0 \mathrm{~mol} \%), \mathrm{Cu}(\mathrm{acac})_{2}(0.0262 \mathrm{~g}, 0.100 \mathrm{mmol}, 10 \mathrm{~mol} \%)$, 4-chlorophenylboronic acid $(0.234 \mathrm{~g}, 1.50 \mathrm{mmol}, 1.50$ equiv $)$, p-toluidine $(0.107 \mathrm{~g}, 1.00 \mathrm{mmol}$, 1.00 equiv), 2,6-lutidine ( $0.231 \mathrm{~mL}, 2.00 \mathrm{mmol}, 2.0$ equiv), and toluene:MeCN (1:1, $3.00 \mathrm{~mL})$. Column chromatography using $20: 1$ hexanes:ethyl acetate provided $\mathbf{3 b}$ as a white solid $(0.198$ g, $0.911 \mathrm{mmol}, 91 \%) .{ }^{1} \mathrm{H}$ NMR $\left(400 \mathrm{MHz}, \mathrm{CDCl}_{3}\right): \delta 7.19(\mathrm{~d}, J=8.5 \mathrm{~Hz}, 1 \mathrm{H}), 7.12(\mathrm{~d}, J=8.6$ $\mathrm{Hz}, 1 \mathrm{H}), 6.99(\mathrm{~d}, J=8.4 \mathrm{~Hz}, 2 \mathrm{H}), 6.93(\mathrm{~d}, J=8.5 \mathrm{~Hz}, 1 \mathrm{H}), 5.58(\mathrm{~s}, 1 \mathrm{H}), 2.33(\mathrm{~s}, 3 \mathrm{H}) .{ }^{13} \mathrm{C} \mathrm{NMR}$ $\left(100 \mathrm{MHz}, \mathrm{CDCl}_{3}\right): \delta 142.83,139.93,131.62,130.08,129.32,124.83,119.34,117.96,20.83$. HRMS (ESI+) Calcd for $\mathrm{C}_{13} \mathrm{H}_{12} \mathrm{CIN}^{+}[\mathrm{M}+\mathrm{H}]^{+}:$218.0731; Found 218.0737.

4-Chloro- $N$-(4-methoxyphenyl)aniline $(\mathbf{3 c}) .{ }^{15}$ The general procedure was followed using $\mathrm{Ru}(\mathrm{bpy})_{3}\left(\mathrm{PF}_{6}\right)_{2}(0.0086 \mathrm{~g}, 0.010 \mathrm{mmol}, 1.0 \mathrm{~mol} \%), \mathrm{Cu}(\mathrm{acac})_{2}(0.0262 \mathrm{~g}, 0.100 \mathrm{mmol}, 10 \mathrm{~mol} \%)$, 4-chlorophenylboronic acid $(0.234 \mathrm{~g}, 1.50 \mathrm{mmol}, 1.50$ equiv), p-anisidine $(0.123 \mathrm{~g}, 1.00 \mathrm{mmol}$, 1.00 equiv), 2,6-lutidine ( $0.231 \mathrm{~mL}, 2.00 \mathrm{mmol}, 2.0$ equiv), and toluene:MeCN (1:1, $3.00 \mathrm{~mL})$. Column chromatography using 20:1 hexanes:ethyl acetate provided $3 \mathbf{c}$ as a white solid $(0.198$ g, $0.928 \mathrm{mmol}, 93 \%) .{ }^{1} \mathrm{H}$ NMR $\left(400 \mathrm{MHz}, \mathrm{CDCl}_{3}\right): \delta 7.15(\mathrm{~d}, J=9.0 \mathrm{~Hz}, 1 \mathrm{H}), 7.05(\mathrm{~d}, J=8.9$ $\mathrm{Hz}, 1 \mathrm{H}), 6.87(\mathrm{~d}, J=9.0 \mathrm{~Hz}, 1 \mathrm{H}), 6.81(\mathrm{~d}, J=8.9 \mathrm{~Hz}, 1 \mathrm{H}), 3.80(\mathrm{~s}, 3 \mathrm{H}) .{ }^{13} \mathrm{C} \mathrm{NMR}(100 \mathrm{MHz}$, $\left.\mathrm{CDCl}_{3}\right): \delta 155.77,144.10,135.35,129.30,124.11,122.72,116.77,114.90,55.72 . \mathrm{HRMS}$ (ESI+) Calcd for $\mathrm{C}_{13} \mathrm{H}_{13} \mathrm{CINO}^{+}[\mathrm{M}+\mathrm{H}]^{+}:$234.0680; Found 234.0673.

4-Chloro- $N$-(4-(trifluoromethyl)phenyl)aniline $(\mathbf{3 d}) .{ }^{4 a}$ The general procedure was followed 
using Ru(bpy $)_{3}\left(\mathrm{PF}_{6}\right)_{2}(0.0086 \mathrm{~g}, 0.010 \mathrm{mmol}, 1.0 \mathrm{~mol} \%), \mathrm{Cu}(\mathrm{acac})_{2}(0.0262 \mathrm{~g}, 0.100 \mathrm{mmol}, 10$ mol\%), 4-chlorophenylboronic acid ( $0.234 \mathrm{~g}, 1.50 \mathrm{mmol}, 1.50$ equiv), 4-(trifluoromethyl)aniline (0.125 mL, $1.00 \mathrm{mmol}, 1.00$ equiv), 2,6-lutidine (0.231 mL, $2.00 \mathrm{mmol}, 2.0$ equiv), and toluene:MeCN (1:1, 3.00 mL). Column chromatography using 20:1 hexanes:ethyl acetate provided 3d as a light yellow oil $(0.212 \mathrm{~g}, 0.783 \mathrm{mmol}, 78 \%) .{ }^{1} \mathrm{H} \mathrm{NMR}\left(400 \mathrm{MHz}, \mathrm{CDCl}_{3}\right): \delta$ $7.48(\mathrm{~d}, J=8.3 \mathrm{~Hz}, 2 \mathrm{H}), 7.28(\mathrm{~d}, J=8.9 \mathrm{~Hz}, 1 \mathrm{H}), 7.08(\mathrm{~d}, J=8.9 \mathrm{~Hz}, 2 \mathrm{H}), 7.02(\mathrm{~d}, J=8.3 \mathrm{~Hz}$, 2H), $5.87(\mathrm{~s}, 1 \mathrm{H}) .{ }^{13} \mathrm{C}$ NMR (100 MHz, $\left.\mathrm{CDCl}_{3}\right): \delta 146.5,139.9,129.7,127.8,126.9$ (q, J = 3.8 $\mathrm{Hz}), 124.8(\mathrm{q}, J=270.6 \mathrm{~Hz}), 123.2,122.3(\mathrm{q}, J=32.7 \mathrm{~Hz}), 121.3,115.7 .{ }^{19} \mathrm{~F}$ NMR $(376 \mathrm{MHz}$, $\left.\mathrm{CDCl}_{3}\right): \delta$-61.16. HRMS (ESI+) Calcd for $\mathrm{C}_{13} \mathrm{H}_{10} \mathrm{ClF}_{3} \mathrm{~N}^{+}[\mathrm{M}+\mathrm{H}]^{+}:$272.0448; Found 272.0451.

$N$-(4-Chlorophenyl)benzo[d][1,3]dioxol-5-amine (4). The general procedure was followed using $\mathrm{Ru}(\mathrm{bpy})_{3}\left(\mathrm{PF}_{6}\right)_{2}(0.0086 \mathrm{~g}, 0.010 \mathrm{mmol}, 1.0 \mathrm{~mol} \%), \mathrm{Cu}(\mathrm{acac})_{2}(0.0262 \mathrm{~g}, 0.100 \mathrm{mmol}, 10$ mol\%), 4-chlorophenylboronic acid $(0.234 \mathrm{~g}, \quad 1.50 \mathrm{mmol}, 1.50$ equiv), 3,4(methylenedioxy)aniline $(0.137 \mathrm{~g}, 1.00 \mathrm{mmol}, 1.00$ equiv), 2,6-lutidine $(0.231 \mathrm{~mL}, 2.00 \mathrm{mmol}$, 2.0 equiv), and toluene:MeCN (1:1, $3.00 \mathrm{~mL})$. Column chromatography using 20:1 hexanes:ethyl acetate provided 4 as a white solid $(0.219 \mathrm{~g}, 0.887 \mathrm{mmol}, 89 \%)$. Mp 148-149 ${ }^{\circ} \mathrm{C}$ IR (thin film): 3413 (m), 3001 (w), 2915 (w), 1656 (w), 1487 (w), 1436 (w), 1406 (w), 1313 (w), 1015 (s), 915 (m) 701 (w), 668 (w). ${ }^{1} \mathrm{H}$ NMR (400 MHz, $\left.\mathrm{CDCl}_{3}\right): \delta 7.16$ (d, J=8.8 Hz, 1H), 6.84 $(\mathrm{d}, J=8.8 \mathrm{~Hz}, 1 \mathrm{H}), 6.74(\mathrm{~d}, J=8.2 \mathrm{~Hz}, 1 \mathrm{H}), 6.66(\mathrm{~d}, J=2.2 \mathrm{~Hz}, 1 \mathrm{H}), 6.53(\mathrm{dd}, J=8.2,2.2 \mathrm{~Hz}$, 1H), $5.94(\mathrm{~s}, 1 \mathrm{H}) .{ }^{13} \mathrm{C} \mathrm{NMR}\left(100 \mathrm{MHz}, \mathrm{CDCl}_{3}\right): \delta 148.44,143.59,143.44,136.87,129.35$, 124.60, 117.34, 113.56, 108.76, 103.01, 101.32. HRMS (ESI+) Calcd for $\mathrm{C}_{13} \mathrm{H}_{11} \mathrm{CINO}_{2}{ }^{+}[\mathrm{M}+\mathrm{H}]^{+}$: 248.0473; Found 248.0469.

1-(4-((4-Chlorophenyl)amino)phenyl)ethan-1-one $\quad(5) .^{4 a}$ The general procedure was 
followed using $\mathrm{Ru}(\mathrm{bpy})_{3}\left(\mathrm{PF}_{6}\right)_{2}(0.0086 \mathrm{~g}, 0.010 \mathrm{mmol}, 1.0 \mathrm{~mol} \%), \mathrm{Cu}(\mathrm{acac})_{2}(0.0262 \mathrm{~g}, 0.100$ mmol, $10 \mathrm{~mol} \%)$, 4-chlorophenylboronic acid (0.234 g, $1.50 \mathrm{mmol}, 1.50$ equiv), 4'aminoacetophenone $(0.135 \mathrm{~g}, 1.00 \mathrm{mmol}, 1.00$ equiv), 2,6-lutidine $(0.231 \mathrm{~mL}, 2.00 \mathrm{mmol}, 2.0$ equiv), and toluene:MeCN (1:1, 3.00 mL). Column chromatography using 20:1 hexanes:ethyl acetate provided 5 as a light yellow solid $(0.167 \mathrm{~g}, 0.681 \mathrm{mmol}, 68 \%) .{ }^{1} \mathrm{H} \mathrm{NMR}(400 \mathrm{MHz}$, $\left.\mathrm{CDCl}_{3}\right): \delta 7.87(\mathrm{~d}, J=8.9 \mathrm{~Hz}, 1 \mathrm{H}), 7.30(\mathrm{~d}, J=8.9 \mathrm{~Hz}, \mathrm{OH}), 7.11(\mathrm{~d}, J=8.9 \mathrm{~Hz}, \mathrm{OH}), 6.97(\mathrm{~d}, J$ $=8.9 \mathrm{~Hz}, 1 \mathrm{H}), 2.53(\mathrm{~s}, 1 \mathrm{H}) .{ }^{13} \mathrm{C}$ NMR $\left(100 \mathrm{MHz}, \mathrm{CDCl}_{3}\right): \delta 196.54,148.03,139.45,130.78$, 129.70, 129.58, 128.31, 121.93, 121.92, 114.78, 26.33. HRMS (ESI+) Calcd for $\mathrm{C}_{14} \mathrm{H}_{13} \mathrm{CINO}^{+}$ $[\mathrm{M}+\mathrm{H}]^{+}:$246.0680; Found 246.0689.

4-Chloro- $N$-(4-chlorophenyl)-3-(trifluoromethyl)aniline (6). The general procedure was followed using Ru(bpy $)_{3}\left(\mathrm{PF}_{6}\right)_{2}(0.0086 \mathrm{~g}, 0.010 \mathrm{mmol}, 1.0 \mathrm{~mol} \%), \mathrm{Cu}(\mathrm{acac})_{2}(0.0262 \mathrm{~g}, 0.100$ mmol, $10 \mathrm{~mol} \%)$, 4-chlorophenylboronic acid (0.234 g, $1.50 \mathrm{mmol}, 1.50$ equiv), 4-chloro-3(trifluoromethyl)aniline (0.195 g, $1.00 \mathrm{mmol}, 1.00$ equiv), 2,6-lutidine $(0.231 \mathrm{~mL}, 2.00 \mathrm{mmol}, 2.0$ equiv), and toluene:MeCN (1:1, $3.00 \mathrm{~mL})$. Column chromatography using 15:1 hexanes:ethyl acetate provided 6 as a light yellow oil $(0.187 \mathrm{~g}, 0.613 \mathrm{mmol}, 61 \%) .{ }^{1} \mathrm{H} \mathrm{NMR}\left(400 \mathrm{MHz}, \mathrm{CDCl}_{3}\right)$ : $\delta 7.19(\mathrm{~d}, J=8.7 \mathrm{~Hz}, 1 \mathrm{H}), 7.12(\mathrm{~d}, J=8.5 \mathrm{~Hz}, 3 \mathrm{H}), 6.94(\mathrm{~d}, J=8.7 \mathrm{~Hz}, 1 \mathrm{H}), 6.86(\mathrm{~d}, J=8.6$ $\mathrm{Hz}, 1 \mathrm{H}), 5.67$ (s, 1H). ${ }^{13} \mathrm{C} N M R\left(100 \mathrm{MHz}, \mathrm{CDCl}_{3}\right): \delta 142.17,140.12,132.39,129.73,127.52$, 126.90, 124.18, 122.73, 121.46, 120.49, 120.41, 118.74, 115.76. $\left.{ }^{19} \mathrm{~F} \mathrm{NMR} \mathrm{(376} \mathrm{MHz,} \mathrm{CDCl}_{3}\right)$ : $\delta$-62.93. HRMS (ESI+) Calcd for $\mathrm{C}_{13} \mathrm{H}_{9} \mathrm{Cl}_{2} \mathrm{~F}_{3} \mathrm{~N}^{+}[\mathrm{M}+\mathrm{H}]^{+}$: 306.0059; Found 306.0064.

Ethyl 3-((4-chlorophenyl)amino)benzoate (7). The general procedure was followed using $\mathrm{Ru}(\mathrm{bpy})_{3}\left(\mathrm{PF}_{6}\right)_{2}(0.0086 \mathrm{~g}, 0.010 \mathrm{mmol}, 1.0 \mathrm{~mol} \%), \mathrm{Cu}(\mathrm{acac})_{2}(0.0262 \mathrm{~g}, 0.100 \mathrm{mmol}, 10 \mathrm{~mol} \%)$, 4-chlorophenylboronic acid $(0.234 \mathrm{~g}, 1.50 \mathrm{mmol}, 1.50$ equiv), ethyl 3-aminobenzoate $(0.165 \mathrm{~g}$, 
$1.00 \mathrm{mmol}, 1.00$ equiv), 2,6-lutidine (0.231 mL, $2.00 \mathrm{mmol}, 2.0$ equiv), and toluene:MeCN (1:1, $3.00 \mathrm{~mL}$ ). Column chromatography using $15: 1$ hexanes:ethyl acetate provided $\mathbf{7}$ as an ivory solid $(0.196 \mathrm{~g}, 0.714 \mathrm{mmol}, 71 \%)$. As an example of a larger-scale reaction with arylamines, the procedure was followed using $\mathrm{Ru}(\mathrm{bpy})_{3}\left(\mathrm{PF}_{6}\right)_{2}(0.034 \mathrm{~g}, 0.040 \mathrm{mmol}, 1.0 \mathrm{~mol} \%), \mathrm{Cu}(\mathrm{acac})_{2}$ (0.105 g, $0.400 \mathrm{mmol}, 10 \mathrm{~mol} \%)$, 4-chlorophenylboronic acid $(0.936 \mathrm{~g}, 6.00 \mathrm{mmol}, 1.50$ equiv), ethyl 3-aminobenzoate $(0.660 \mathrm{~g}, 4.00 \mathrm{mmol}, 1.00$ equiv), 2,6-lutidine $(0.924 \mathrm{~mL}, 8.00 \mathrm{mmol}$, 2.0 equiv), and toluene:MeCN $(1: 1,12.00 \mathrm{~mL})$. Column chromatography using 15:1 hexanes:ethyl acetate provided 7 as an ivory solid $(0.758 \mathrm{~g}, 2.76 \mathrm{mmol}, 70 \%)$. Mp 184-185 ${ }^{\circ} \mathrm{C}$ IR (thin film): 3013 (w), 2159 (w), 2121 (w), 2072 (w), 1713 (w), 1663 (w), 1588 (w), 1491 (w), $952(\mathrm{~m}) .{ }^{1} \mathrm{H}$ NMR $\left(400 \mathrm{MHz}, \mathrm{CDCl}_{3}\right): \delta 7.71-7.67(\mathrm{~m}, \mathrm{OH}), 7.60(\mathrm{dt}, J=7.6,1.3 \mathrm{~Hz}, \mathrm{OH}), 7.32$ (t, $J=7.9 \mathrm{~Hz}, \mathrm{OH}), 7.27-7.19(\mathrm{~m}, 1 \mathrm{H}), 7.01(\mathrm{~d}, J=8.9 \mathrm{~Hz}, 1 \mathrm{H}), 4.37(\mathrm{q}, J=7.1 \mathrm{~Hz}, 1 \mathrm{H}), 1.38$ (t, $J=7.1 \mathrm{~Hz}, 1 \mathrm{H}) .{ }^{13} \mathrm{C}$ NMR $\left(100 \mathrm{MHz}, \mathrm{CDCl}_{3}\right): \delta 166.61,143.18,141.31,131.95,129.55$, 129.53, 126.41, 122.38, 121.78, 119.50, 118.63, 61.21, 14.46. HRMS (ESI+) Calcd for $\mathrm{C}_{15} \mathrm{H}_{15} \mathrm{CINO}_{2}^{+}[\mathrm{M}+\mathrm{H}]^{+}:$276.0786; Found 276.0780.

$\mathrm{N}$-(4-Chlorophenyl)naphthalen-1-amine (8). ${ }^{16}$ The general procedure was followed using $\mathrm{Ru}(\mathrm{bpy})_{3}\left(\mathrm{PF}_{6}\right)_{2}(0.0086 \mathrm{~g}, 0.010 \mathrm{mmol}, 1.0 \mathrm{~mol} \%), \mathrm{Cu}(\mathrm{acac})_{2}(0.0262 \mathrm{~g}, 0.100 \mathrm{mmol}, 10 \mathrm{~mol} \%)$, 4-chlorophenylboronic acid (0.234 g, $1.50 \mathrm{mmol}, 1.50$ equiv), naphthalen-1-amine (0.143 g, $1.00 \mathrm{mmol}, 1.00$ equiv), 2,6-lutidine ( $0.231 \mathrm{~mL}, 2.00 \mathrm{mmol}, 2.0$ equiv), and toluene:MeCN (1:1, $3.00 \mathrm{~mL}$ ). Column chromatography using $20: 1$ hexanes:ethyl acetate provided 8 as an ivory solid (0.163 g, $0.643 \mathrm{mmol}, 64 \%) .{ }^{1} \mathrm{H}$ NMR $\left(400 \mathrm{MHz}, \mathrm{CDCl}_{3}\right): \delta 8.02-7.95(\mathrm{~m}, 1 \mathrm{H}), 7.88$ (dd, $J=7.4,2.1 \mathrm{~Hz}, 1 \mathrm{H}), 7.61(\mathrm{~d}, J=8.1 \mathrm{~Hz}, 1 \mathrm{H}), 7.55-7.45(\mathrm{~m}, 2 \mathrm{H}), 7.44-7.37(\mathrm{~m}, 1 \mathrm{H}), 7.33$ (dd, $J=7.4,1.2 \mathrm{~Hz}, 1 \mathrm{H}), 7.24-7.15(\mathrm{~m}, 2 \mathrm{H}), 6.91-6.85(\mathrm{~m}, 2 \mathrm{H}) .{ }^{13} \mathrm{C} \mathrm{NMR}\left(100 \mathrm{MHz}, \mathrm{CDCl}_{3}\right)$ : 
$\delta 143.82,138.39,134.85,129.38,128.73,128.09,126.38,126.12,126.02,125.04,123.82$, 121.97, 118.32, 116.90. HRMS (ESI+) Calcd for $\mathrm{C}_{16} \mathrm{H}_{13} \mathrm{CIN}^{+}[\mathrm{M}+\mathrm{H}]^{+}$: 253.0731; Found 253.0735 .

2-(4-((4-Chlorophenyl)amino)phenyl)ethan-1-ol (9). The general procedure was followed using $\mathrm{Ru}(\mathrm{bpy})_{3}\left(\mathrm{PF}_{6}\right)_{2}(0.0086 \mathrm{~g}, 0.010 \mathrm{mmol}, 1.0 \mathrm{~mol} \%), \mathrm{Cu}(\mathrm{acac})_{2}(0.0262 \mathrm{~g}, 0.100 \mathrm{mmol}, 10$ mol\%), 4-chlorophenylboronic acid (0.234 g, $1.50 \mathrm{mmol}, 1.50$ equiv), 2-(4-aminophenyl)ethan1-ol (0.137 g, $1.00 \mathrm{mmol}, 1.00$ equiv), 2,6-lutidine (0.231 mL, $2.00 \mathrm{mmol}, 2.0$ equiv), and toluene:MeCN (1:1, $3.00 \mathrm{~mL})$. Column chromatography using 10:1 hexanes:ethyl acetate provided 9 as an ivory solid $(0.207 \mathrm{~g}, 0.838 \mathrm{mmol}, 84 \%) . \mathrm{Mp} 152-154{ }^{\circ} \mathrm{C}$ IR (thin film): 3582 (br), 3023 (w), 2982 (m), 1574 (w), 1499 (w), 1337 (w), 1084 (w), 879 (w). ${ }^{1} \mathrm{H}$ NMR (400 MHz, $\left.\mathrm{CDCl}_{3}\right): \delta 7.20(\mathrm{~d}, J=8.9 \mathrm{~Hz}, 1 \mathrm{H}), 7.14(\mathrm{~d}, J=8.5 \mathrm{~Hz}, 1 \mathrm{H}), 7.00(\mathrm{~d}, J=8.5 \mathrm{~Hz}, 1 \mathrm{H}), 6.95(\mathrm{~d}, J$ $=8.9 \mathrm{~Hz}, 1 \mathrm{H}), 3.84(\mathrm{t}, J=6.6 \mathrm{~Hz}, 1 \mathrm{H}), 2.82(\mathrm{t}, J=6.6 \mathrm{~Hz}, 1 \mathrm{H}) \cdot{ }^{13} \mathrm{C} \mathrm{NMR}\left(100 \mathrm{MHz}, \mathrm{CDCl}_{3}\right): \delta$ 142.24, 141.10, 131.65, 130.04, 129.29, 125.14, 118.72, 118.42, 63.77, 38.48. HRMS (ESI+) Calcd for $\mathrm{C}_{14} \mathrm{H}_{15} \mathrm{CINO}^{+}[\mathrm{M}+\mathrm{H}]^{+}$: 248.0837; Found 248.0831.

Diphenylamine (10). ${ }^{6 c}$ The general procedure was followed using $\mathrm{Ru}(\mathrm{bpy})_{3}\left(\mathrm{PF}_{6}\right)_{2}(0.0086 \mathrm{~g}$, $0.010 \mathrm{mmol}, 1.0 \mathrm{~mol} \%), \mathrm{Cu}(\mathrm{acac})_{2}(0.0262 \mathrm{~g}, 0.100 \mathrm{mmol}, 10 \mathrm{~mol} \%)$, phenylboronic acid (0.183 g, $1.50 \mathrm{mmol}, 1.50$ equiv), aniline ( $0.091 \mathrm{~mL}, 1.00 \mathrm{mmol}, 1.00$ equiv), 2,6-lutidine (0.231 $\mathrm{mL}, 2.00 \mathrm{mmol}, 2.0$ equiv), and toluene:MeCN (1:1, $3.00 \mathrm{~mL})$. Column chromatography using 20:1 hexanes:ethyl acetate provided 10 as a white solid $(0.152 \mathrm{~g}, 0.897 \mathrm{mmol}, 90 \%) .{ }^{1} \mathrm{H}$ NMR $\left(400 \mathrm{MHz}, \mathrm{CDCl}_{3}\right): \delta 7.35-7.24(\mathrm{~m}, 2 \mathrm{H}), 7.09(\mathrm{~d}, J=8.6 \mathrm{~Hz}, 3 \mathrm{H}), 6.95(\mathrm{ddd}, J=7.3,6.7,1.1$ $\mathrm{Hz}, 1 \mathrm{H}), 5.70(\mathrm{~s}, 1 \mathrm{H}) .{ }^{13} \mathrm{C}$ NMR $\left(100 \mathrm{MHz}, \mathrm{CDCl}_{3}\right): \delta 143.25,129.47,121.13,117.95 . \mathrm{HRMS}$ (ESI+) Calcd for $\mathrm{C}_{12} \mathrm{H}_{12} \mathrm{~N}^{+}[\mathrm{M}+\mathrm{H}]^{+}:$170.0964; Found 170.0959 . 
4-Methyl- $N$-phenylaniline (11) ${ }^{6 c}$ The general procedure was followed using $\mathrm{Ru}(\mathrm{bpy})_{3}\left(\mathrm{PF}_{6}\right)_{2}$ (0.0086 g, $0.010 \mathrm{mmol}, 1.0 \mathrm{~mol} \%), \mathrm{Cu}(\mathrm{acac})_{2}(0.0262 \mathrm{~g}, 0.100 \mathrm{mmol}, 10 \mathrm{~mol} \%), p$-tolylboronic acid $(0.204 \mathrm{~g}, 1.50 \mathrm{mmol}, 1.50$ equiv), aniline (0.091 mL, $1.00 \mathrm{mmol}, 1.00$ equiv), 2,6-lutidine (0.231 mL, $2.00 \mathrm{mmol}, 2.0$ equiv), and toluene:MeCN (1:1, $3.00 \mathrm{~mL})$. Column chromatography using 20:1 hexanes:ethyl acetate provided 11 as a white solid $(0.156 \mathrm{~g}, 0.854 \mathrm{mmol}, 85 \%) .{ }^{1} \mathrm{H}$ NMR (400 MHz, $\left.\mathrm{CDCl}_{3}\right): \delta 7.28-7.22(\mathrm{~m}, 2 \mathrm{H}), 7.12-7.08(\mathrm{~m}, 1 \mathrm{H}), 7.06-6.99(\mathrm{~m}, 3 \mathrm{H}), 6.92$ $-6.86(\mathrm{~m}, 1 \mathrm{H}), 5.61(\mathrm{~s}, 1 \mathrm{H}), 2.32(\mathrm{~s}, 4 \mathrm{H}) .{ }^{13} \mathrm{C} \mathrm{NMR}\left(100 \mathrm{MHz}, \mathrm{CDCl}_{3}\right): \delta$ 144.09, 140.42, 131.07, 129.99, 129.43, 120.43, 119.04, 117.00, 20.83. HRMS (ESI+) Calcd for $\mathrm{C}_{13} \mathrm{H}_{14} \mathrm{~N}^{+}$ $[\mathrm{M}+\mathrm{H}]^{+}:$184.1121; Found 184.1118.

$\mathrm{N}$-Phenyl-4-(trifluoromethyl)aniline (12). ${ }^{17}$ The general procedure was followed using $\mathrm{Ru}(\mathrm{bpy})_{3}\left(\mathrm{PF}_{6}\right)_{2}(0.0086 \mathrm{~g}, 0.010 \mathrm{mmol}, 1.0 \mathrm{~mol} \%), \mathrm{Cu}(\mathrm{acac})_{2}(0.0262 \mathrm{~g}, 0.100 \mathrm{mmol}, 10 \mathrm{~mol} \%)$, (4-(trifluoromethyl)phenyl)boronic acid (0.285 g, $1.50 \mathrm{mmol}, 1.50$ equiv), aniline $(0.091 \mathrm{~mL}$, $1.00 \mathrm{mmol}, 1.00$ equiv), 2,6-lutidine ( $0.231 \mathrm{~mL}, 2.00 \mathrm{mmol}, 2.0$ equiv), and toluene:MeCN (1:1, 3.00 mL). Column chromatography using 20:1 hexanes:ethyl acetate provided 12 as a white solid (0.194 g, $0.821 \mathrm{mmol}, 82 \%) .{ }^{1} \mathrm{H}$ NMR $\left(400 \mathrm{MHz}, \mathrm{CDCl}_{3}\right): \delta 7.47(\mathrm{~d}, J=8.5 \mathrm{~Hz}, 1 \mathrm{H}), 7.38$ $-7.28(\mathrm{~m}, 1 \mathrm{H}), 7.15(\mathrm{~d}, J=8.4 \mathrm{~Hz}, 1 \mathrm{H}), 7.05(\mathrm{t}, J=8.2 \mathrm{~Hz}, 2 \mathrm{H}) .{ }^{13} \mathrm{C} \mathrm{NMR}\left(100 \mathrm{MHz}, \mathrm{CDCl}_{3}\right): \delta$ $146.91,141.29,129.68,129.67,127.78,126.89,126.85,126.82,126.78,126.09,123.40$, $123.09,123.07,121.97,121.65,120.19,120.17,119.21,115.48,115.45 .{ }^{19} \mathrm{~F}$ NMR $(376 \mathrm{MHz}$, $\left.\mathrm{CDCl}_{3}\right): \delta$-62.77. HRMS (ESI+) Calcd for $\mathrm{C}_{13} \mathrm{H}_{11} \mathrm{~F}_{3} \mathrm{~N}^{+}[\mathrm{M}+\mathrm{H}]^{+}:$238.0838; Found 238.0832.

4-Bromo- $N$-phenylaniline (13). ${ }^{4 a}$ The general procedure was followed using $R u(b p y)_{3}\left(\mathrm{PF}_{6}\right)_{2}$ (0.0086 g, $0.010 \mathrm{mmol}, 1.0 \mathrm{~mol} \%), \mathrm{Cu}(\mathrm{acac})_{2}(0.0262 \mathrm{~g}, 0.100 \mathrm{mmol}, 10 \mathrm{~mol} \%)$, (4bromophenyl)boronic acid $(0.299 \mathrm{~g}, 1.50 \mathrm{mmol}, 1.50$ equiv), aniline $(0.091 \mathrm{~mL}, 1.00 \mathrm{mmol}$, 
1.00 equiv), 2,6-lutidine ( $0.231 \mathrm{~mL}, 2.00 \mathrm{mmol}, 2.0$ equiv), and toluene:MeCN (1:1, $3.00 \mathrm{~mL})$. Column chromatography using 20:1 hexanes:ethyl acetate provided 13 as a tan solid $(0.184 \mathrm{~g}$, $0.744 \mathrm{mmol}, 74 \%) .{ }^{1} \mathrm{H}$ NMR $\left(400 \mathrm{MHz}, \mathrm{CDCl}_{3}\right): \delta 7.34(\mathrm{~d}, J=8.9 \mathrm{~Hz}, 1 \mathrm{H}), 7.28(\mathrm{dd}, J=8.5$, $7.4 \mathrm{~Hz}, 1 \mathrm{H}), 7.06(\mathrm{dd}, J=8.6,1.0 \mathrm{~Hz}, 1 \mathrm{H}), 7.01-6.89(\mathrm{~m}, 1 \mathrm{H}), 5.67(\mathrm{~s}, 1 \mathrm{H}) .{ }^{13} \mathrm{C}$ NMR $(100$ $\left.\mathrm{MHz}, \mathrm{CDCl}_{3}\right): \delta 142.57,142.55,132.32,129.59,121.81,119.17,118.45,112.77 . \mathrm{HRMS}(\mathrm{ESI}+)$ Calcd for $\mathrm{C}_{12} \mathrm{H}_{11} \mathrm{BrN}^{+}[\mathrm{M}+\mathrm{H}]^{+}$: 248.0069; Found 248.0073.

$\mathrm{N}$-(4-Chlorophenyl)-2-methylaniline (14). ${ }^{4 a}$ The general procedure was followed using $\mathrm{Ru}(\mathrm{bpy})_{3}\left(\mathrm{PF}_{6}\right)_{2}(0.0086 \mathrm{~g}, 0.010 \mathrm{mmol}, 1.0 \mathrm{~mol} \%), \mathrm{Cu}(\mathrm{acac})_{2}(0.0262 \mathrm{~g}, 0.100 \mathrm{mmol}, 10 \mathrm{~mol} \%)$, o-tolylboronic acid (0.204 g, $1.50 \mathrm{mmol}, 1.50$ equiv), 4-chloroaniline $(0.127 \mathrm{~g}, 1.00 \mathrm{mmol}, 1.00$ equiv), 2,6-lutidine (0.231 mL, $2.00 \mathrm{mmol}, 2.0$ equiv), and toluene:MeCN (1:1, $3.00 \mathrm{~mL})$. Column chromatography using 20:1 hexanes:ethyl acetate provided 14 as a tan oil $(0.137 \mathrm{~g}$, $0.631 \mathrm{mmol}, 63 \%) .{ }^{1} \mathrm{H}$ NMR $\left(400 \mathrm{MHz}, \mathrm{CDCl}_{3}\right): \delta 7.19(\mathrm{~d}, J=9.0 \mathrm{~Hz}, 2 \mathrm{H}), 7.24-7.11(\mathrm{~m}, 3 \mathrm{H})$, $6.98(\mathrm{td}, J=7.1,1.7 \mathrm{~Hz}, 1 \mathrm{H}), 6.85(\mathrm{~d}, J=8.9 \mathrm{~Hz}, 2 \mathrm{H}), 4.62(\mathrm{~s}, 1 \mathrm{H}), 2.25(\mathrm{~s}, 3 \mathrm{H}) .{ }^{13} \mathrm{C}$ NMR $(100$ $\left.\mathrm{MHz}, \mathrm{CDCl}_{3}\right): \delta 142.97,140.82,131.21,129.34,129.10,127.00,125.02,122.80,119.61$, 118.40, 18.01. HRMS (ESI+) Calcd for $\mathrm{C}_{13} \mathrm{H}_{13} \mathrm{CIN}^{+}[\mathrm{M}+\mathrm{H}]^{+}$: 218.0731; Found 218.0733.

Bis(4-chlorophenyl)amine (15). ${ }^{4 a}$ The general procedure was followed using $\mathrm{Ru}(\mathrm{bpy})_{3}\left(\mathrm{PF}_{6}\right)_{2}$ $(0.0086 \mathrm{~g}, 0.010 \mathrm{mmol}, 1.0 \mathrm{~mol} \%), \mathrm{Cu}(\mathrm{acac})_{2}(0.0262 \mathrm{~g}, 0.100 \mathrm{mmol}, 10 \mathrm{~mol} \%), 4-$ chloroboronic acid (0.234 g, $1.50 \mathrm{mmol}, 1.50$ equiv), 4-chloroaniline $(0.127 \mathrm{~g}, 1.00 \mathrm{mmol}, 1.00$ equiv), 2,6-lutidine (0.231 mL, $2.00 \mathrm{mmol}, 2.0$ equiv), and toluene:MeCN (1:1, $3.00 \mathrm{~mL})$. Column chromatography using 20:1 hexanes:ethyl acetate provided 15 as a tan solid $(0.164 \mathrm{~g}$, $0.694 \mathrm{mmol}, 69 \%) .{ }^{1} \mathrm{H}$ NMR $\left(400 \mathrm{MHz}, \mathrm{CDCl}_{3}\right): \delta 7.22(\mathrm{~d}, J=8.8 \mathrm{~Hz}, 4 \mathrm{H}), 6.96(\mathrm{~d}, J=8.8 \mathrm{~Hz}$, 4H). ${ }^{13} \mathrm{C}$ NMR $\left(100 \mathrm{MHz}, \mathrm{CDCl}_{3}\right): \delta 141.54,129.54,126.23,119.27 . \mathrm{HRMS}(\mathrm{ESI}+)$ Calcd for 
$\mathrm{C}_{12} \mathrm{H}_{10} \mathrm{Cl}_{2} \mathrm{~N}^{+}[\mathrm{M}+\mathrm{H}]^{+}:$238.0185; Found 238.0188.

Methyl 4-((4-chlorophenyl)amino)benzoate (16). The general procedure was followed using $\mathrm{Ru}(\mathrm{bpy})_{3}\left(\mathrm{PF}_{6}\right)_{2}(0.0086 \mathrm{~g}, 0.010 \mathrm{mmol}, 1.0 \mathrm{~mol} \%), \mathrm{Cu}(\mathrm{acac})_{2}(0.0262 \mathrm{~g}, 0.100 \mathrm{mmol}, 10 \mathrm{~mol} \%)$, (4-(methoxycarbonyl)phenyl)boronic acid $(0.270 \mathrm{~g}, 1.50 \mathrm{mmol}, 1.50$ equiv), 4-chloroaniline (0.127 g, $1.00 \mathrm{mmol}, 1.00$ equiv), 2,6-lutidine $(0.231 \mathrm{~mL}, 2.00 \mathrm{mmol}, 2.0$ equiv), and toluene:MeCN (1:1, 3.00 mL). Column chromatography using 20:1 hexanes:ethyl acetate provided 16 as a tan solid $(0.212 \mathrm{~g}, 0.811 \mathrm{mmol}, 81 \%)$. Mp 143-144 ${ }^{\circ} \mathrm{C} \mathrm{IR}$ (thin film): 2990 (w), $2010(w), 1704$ (w), $1278(\mathrm{~m}), 1173(\mathrm{w}), 1276(\mathrm{w}), 917(\mathrm{~m}) .{ }^{1} \mathrm{H}$ NMR $\left(400 \mathrm{MHz}, \mathrm{CDCl}_{3}\right): \delta 7.92$ $(\mathrm{d}, J=8.9 \mathrm{~Hz}, 2 \mathrm{H}), 7.29(\mathrm{~d}, J=8.9 \mathrm{~Hz}, 2 \mathrm{H}), 7.10(\mathrm{~d}, J=8.9 \mathrm{~Hz}, 2 \mathrm{H}), 6.96(\mathrm{~d}, J=8.9 \mathrm{~Hz}, 2 \mathrm{H})$, $5.99(\mathrm{~s}, 1 \mathrm{H}), 3.88(\mathrm{~s}, 3 \mathrm{H}) .{ }^{13} \mathrm{C}$ NMR $\left(100 \mathrm{MHz}, \mathrm{CDCl}_{3}\right): \delta 166.99,147.73,139.68,131.67$, $129.67,128.05,121.78,121.68,114.94,51.92$. HRMS (ESI+) Calcd for $\mathrm{C}_{14} \mathrm{H}_{13} \mathrm{CINO}_{2}{ }^{+}[\mathrm{M}+\mathrm{H}]^{+}$: 262.0629; Found 262.0631.

6-Chloro- $N$-(4-chlorophenyl)pyridin-3-amine (17). The general procedure was followed using $\mathrm{Ru}(\mathrm{bpy})_{3}\left(\mathrm{PF}_{6}\right)_{2}(0.0086 \mathrm{~g}, 0.010 \mathrm{mmol}, 1.0 \mathrm{~mol} \%), \mathrm{Cu}(\mathrm{acac})_{2}(0.0262 \mathrm{~g}, 0.100 \mathrm{mmol}, 10$ mol\%), (6-chloropyridin-3-yl)boronic acid (0.236 g, $1.50 \mathrm{mmol}, 1.50$ equiv), 4-chloroaniline (0.127 g, $1.00 \mathrm{mmol}, 1.00$ equiv), 2,6-lutidine (0.231 $\mathrm{mL}, 2.00 \mathrm{mmol}, 2.0$ equiv), and toluene:MeCN (1:1, 3.00 mL). Column chromatography using 15:1 hexanes:ethyl acetate provided 17 as an ivory oil $(0.173 \mathrm{~g}, 0.727 \mathrm{mmol}, 73 \%)$. IR (thin film): 3447 (m), 3045 (w), 1651 (w), $1493(w), 1464(w), 1276(w), 1276(w), 728(m) .{ }^{1} \mathrm{H}$ NMR (400 MHz, CDCl $): \delta 8.10(s$, 1H), $7.34(\mathrm{dd}, J=8.6,2.9 \mathrm{~Hz}, 2 \mathrm{H}), 7.25(\mathrm{~d}, J=8.8 \mathrm{~Hz}, 2 \mathrm{H}), 7.19(\mathrm{~d}, J=8.6 \mathrm{~Hz}, 2 \mathrm{H}), 6.98(\mathrm{~d}, J$ $=8.8 \mathrm{~Hz}, 2 \mathrm{H}), 5.80(\mathrm{~s}, 1 \mathrm{H}) .{ }^{13} \mathrm{C}$ NMR $\left(100 \mathrm{MHz}, \mathrm{CDCl}_{3}\right): \delta 142.70,140.31,139.29,138.91$, 129.78, 127.47, 126.98, 124.49, 119.90, 119.87. HRMS (ESI+) Calcd for $\mathrm{C}_{11} \mathrm{H}_{9} \mathrm{Cl}_{2} \mathrm{O}_{2}{ }^{+}[\mathrm{M}+\mathrm{H}]^{+}$: 
239.0137; Found 239.0142.

4-Chloro- $N$-hexylaniline (18). ${ }^{18}$ The general procedure was followed using $\mathrm{Ru}(\mathrm{bpy})_{3}\left(\mathrm{PF}_{6}\right)_{2}$ $(0.0086 \mathrm{~g}, 0.010 \mathrm{mmol}, 1.0 \mathrm{~mol} \%), \mathrm{Cu}(\mathrm{acac})_{2}(0.0262 \mathrm{~g}, 0.100 \mathrm{mmol}, 10 \mathrm{~mol} \%), 4-$ chloroboronic acid $(0.234 \mathrm{~g}, 1.50 \mathrm{mmol}, 1.50$ equiv), hexylamine $(0.132 \mathrm{~mL}, 1.00 \mathrm{mmol}, 1.00$ equiv), 2,6-lutidine (0.231 mL, $2.00 \mathrm{mmol}, 2.0$ equiv), and toluene:MeCN (1:1, $3.00 \mathrm{~mL})$. Column chromatography using $20: 1$ hexanes:ethyl acetate provided $\mathbf{1 8}$ as a light yellow oil (0.166 g, $0.787 \mathrm{mmol}, 79 \%) .{ }^{1} \mathrm{H}$ NMR $\left(400 \mathrm{MHz}, \mathrm{CDCl}_{3}\right): \delta 7.12(\mathrm{~d}, J=8.8 \mathrm{~Hz}, 2 \mathrm{H}), 6.57(\mathrm{~d}, J$ $=8.8 \mathrm{~Hz}, 2 \mathrm{H}), 3.79(\mathrm{~s}, 1 \mathrm{H}), 3.07(\mathrm{t}, J=7.2 \mathrm{~Hz}, 2 \mathrm{H}), 1.61(\mathrm{p}, J=7.1 \mathrm{~Hz}, 2 \mathrm{H}), 1.45-1.23(\mathrm{~m}$, $6 \mathrm{H}), 0.95-0.87(\mathrm{~m}, 3 \mathrm{H}) .{ }^{13} \mathrm{C} \mathrm{NMR}\left(100 \mathrm{MHz}, \mathrm{CDCl}_{3}\right): \delta 146.37,129.22,122.62,114.56,44.88$, 31.72, 29.29, 26.90, 22.73, 14.16. HRMS (ESI+) Calcd for $\mathrm{C}_{12} \mathrm{H}_{19} \mathrm{ClN}^{+}[\mathrm{M}+\mathrm{H}]^{+}:$212.1201; Found 212.1205.

4-Chloro- $N$-isopropylaniline (19). ${ }^{19}$ The general procedure was followed using $\mathrm{Ru}(\mathrm{bpy})_{3}\left(\mathrm{PF}_{6}\right)_{2}(0.0086 \mathrm{~g}, 0.010 \mathrm{mmol}, 1.0 \mathrm{~mol} \%), \mathrm{Cu}(\mathrm{acac})_{2}(0.0262 \mathrm{~g}, 0.100 \mathrm{mmol}, 10 \mathrm{~mol} \%)$, 4-chloroboronic acid (0.234 g, $1.50 \mathrm{mmol}, 1.50$ equiv), isopropylamine (0.086 mL, $1.00 \mathrm{mmol}$, 1.00 equiv), 2,6-lutidine ( $0.231 \mathrm{~mL}, 2.00 \mathrm{mmol}, 2.0$ equiv), and toluene:MeCN (1:1, $3.00 \mathrm{~mL})$. Column chromatography using 20:1 hexanes:ethyl acetate provided 19 as a light yellow oil (0.125 g, $0.742 \mathrm{mmol}, 74 \%) .{ }^{1} \mathrm{H}$ NMR $\left(400 \mathrm{MHz}, \mathrm{CDCl}_{3}\right): \delta 7.31$ (d, J=8.8 Hz, 2H), 7.24 (d, J $=8.8 \mathrm{~Hz}, 2 \mathrm{H}), 3.53$ (hept, $J=6.5 \mathrm{~Hz}, 1 \mathrm{H}), 1.27(\mathrm{~d}, J=6.5 \mathrm{~Hz}, 6 \mathrm{H}) \cdot{ }^{13} \mathrm{C} \mathrm{NMR}\left(100 \mathrm{MHz}, \mathrm{CDCl}_{3}\right)$ : $\delta 134.31,134.27,130.04,124.36,54.90,19.26$. HRMS (ESI+) Calcd for $\mathrm{C}_{9} \mathrm{H}_{13} \mathrm{CIN}^{+}[\mathrm{M}+\mathrm{H}]^{+}$: 170.0731; Found 170.0727.

4-Chloro- $N$-cyclohexylaniline (20). ${ }^{20}$ The general procedure was followed using $\mathrm{Ru}(\mathrm{bpy})_{3}\left(\mathrm{PF}_{6}\right)_{2}(0.0086 \mathrm{~g}, 0.010 \mathrm{mmol}, 1.0 \mathrm{~mol} \%), \mathrm{Cu}(\mathrm{acac})_{2}(0.0262 \mathrm{~g}, 0.100 \mathrm{mmol}, 10 \mathrm{~mol} \%)$, 
4-chloroboronic acid (0.234 g, $1.50 \mathrm{mmol}, 1.50$ equiv), cyclohexylamine $(0.114 \mathrm{~mL}, 1.00 \mathrm{mmol}$, 1.00 equiv), 2,6-lutidine (0.231 mL, $2.00 \mathrm{mmol}, 2.0$ equiv), and toluene:MeCN (1:1, $3.00 \mathrm{~mL})$. Column chromatography using $20: 1$ hexanes:ethyl acetate provided 20 as a light yellow oil $(0.158 \mathrm{~g}, 0.758 \mathrm{mmol}, 76 \%)$. As an example of a larger-scale reaction with alkylamines, the procedure was followed using $\mathrm{Ru}(\mathrm{bpy})_{3}\left(\mathrm{PF}_{6}\right)_{2}(0.0580 \mathrm{~g}, 0.0675 \mathrm{mmol}, 1.0 \mathrm{~mol} \%), \mathrm{Cu}(\mathrm{acac})_{2}$ (0.177 g, $0.675 \mathrm{mmol}, 10 \mathrm{~mol} \%)$, 4-chloroboronic acid (1.58 g, $10.1 \mathrm{mmol}, 1.50$ equiv), cyclohexylamine (1.05 mL, $6.75 \mathrm{mmol}, 1.00$ equiv), 2,6-lutidine (1.56 mL, $13.5 \mathrm{mmol}, 2.0$ equiv), and toluene:MeCN (1:1, $20.0 \mathrm{~mL})$. Column chromatography using 20:1 hexanes:ethyl acetate provided 20 as a light yellow oil $(1.09 \mathrm{~g}, 5.22 \mathrm{mmol}, 77 \%) .{ }^{1} \mathrm{H} \mathrm{NMR}\left(400 \mathrm{MHz}, \mathrm{CDCl}_{3}\right)$ : $\delta 7.37-7.27(\mathrm{~m}, 4 \mathrm{H}), 3.15(\mathrm{tt}, J=11.7,3.8 \mathrm{~Hz}, 1 \mathrm{H}), 1.91(\mathrm{~d}, J=12.1 \mathrm{~Hz}, 2 \mathrm{H}), 1.82-1.73(\mathrm{~m}$, 2H), $1.63(\mathrm{~d}, J=8.4 \mathrm{~Hz}, 1 \mathrm{H}), 1.39(\mathrm{q}, J=11.8 \mathrm{~Hz}, 2 \mathrm{H}), 1.16(\mathrm{t}, J=10.9 \mathrm{~Hz}, 3 \mathrm{H}) .{ }^{13} \mathrm{C}$ NMR $\left(100 \mathrm{MHz}, \mathrm{CDCl}_{3}\right): \delta 135.25,132.90,130.03,125.23,62.43,29.13,24.89,24.62 . \mathrm{HRMS}$ (ESI+) Calcd for $\mathrm{C}_{12} \mathrm{H}_{17} \mathrm{CIN}^{+}[\mathrm{M}+\mathrm{H}]^{+}:$: 210.1044; Found 210.1047.

$\mathrm{N}$-Benzyl-4-chloroaniline (21). ${ }^{21}$ The general procedure was followed using $\mathrm{Ru}(\mathrm{bpy})_{3}\left(\mathrm{PF}_{6}\right)_{2}$ (0.0086 g, $0.010 \mathrm{mmol}, 1.0 \mathrm{~mol} \%), \mathrm{Cu}(\mathrm{acac})_{2}(0.0262 \mathrm{~g}, 0.100 \mathrm{mmol}, 10 \mathrm{~mol} \%), 4-$ chloroboronic acid $(0.234 \mathrm{~g}, 1.50 \mathrm{mmol}, 1.50$ equiv), benzylamine $(0.109 \mathrm{~mL}, 1.00 \mathrm{mmol}, 1.00$ equiv), 2,6-lutidine (0.231 mL, $2.00 \mathrm{mmol}, 2.0$ equiv), and toluene:MeCN (1:1, $3.00 \mathrm{~mL})$. Column chromatography using 20:1 hexanes:ethyl acetate provided $\mathbf{2 1}$ as a light yellow oil (0.178 g, $0.823 \mathrm{mmol}, 82 \%) .{ }^{1} \mathrm{H}$ NMR $\left(400 \mathrm{MHz}, \mathrm{CDCl}_{3}\right): \delta 8.17(\mathrm{~s}, 1 \mathrm{H}), 7.34-7.25(\mathrm{~m}, 5 \mathrm{H})$, $7.20(\mathrm{~d}, J=8.9 \mathrm{~Hz}, 2 \mathrm{H}), 6.90(\mathrm{~d}, J=8.9 \mathrm{~Hz}, 2 \mathrm{H}), 4.28(\mathrm{~s}, 2 \mathrm{H}) .{ }^{13} \mathrm{C} \mathrm{NMR}\left(100 \mathrm{MHz}, \mathrm{CDCl}_{3}\right): \delta$ 139.99, 134.09, 129.68, 129.15, 128.91, 128.69, 119.74, 52.74. HRMS (ESI+) Calcd for $\mathrm{C}_{13} \mathrm{H}_{13} \mathrm{CIN}^{+}[\mathrm{M}+\mathrm{H}]^{+}:$218.0731; Found 218.0736. 
4-Chloro- $N$-(2-(cyclohex-1-en-1-yl)ethyl)aniline $(\mathbf{2 2}) .^{22}$ The general procedure was followed using $\mathrm{Ru}(\mathrm{bpy})_{3}\left(\mathrm{PF}_{6}\right)_{2}(0.0086 \mathrm{~g}, 0.010 \mathrm{mmol}, 1.0 \mathrm{~mol} \%), \mathrm{Cu}(\mathrm{acac})_{2}(0.0262 \mathrm{~g}, 0.100 \mathrm{mmol}, 10$ mol\%), 4-chloroboronic acid (0.234 g, $1.50 \mathrm{mmol}, 1.50$ equiv), 2-(cyclohex-1-en-1-yl)ethan-1amine (0.139 mL, $1.00 \mathrm{mmol}, 1.00$ equiv), 2,6-lutidine $(0.231 \mathrm{~mL}, 2.00 \mathrm{mmol}, 2.0$ equiv), and toluene:MeCN (1:1, 3.00 mL). Column chromatography using 20:1 hexanes:ethyl acetate provided 22 as a light yellow oil $(0.163 \mathrm{~g}, 0.694 \mathrm{mmol}, 69 \%) .{ }^{1} \mathrm{H} \mathrm{NMR}\left(400 \mathrm{MHz}, \mathrm{CDCl}_{3}\right): \delta$ $8.27(\mathrm{~s}, 1 \mathrm{H}), 7.31(\mathrm{~d}, J=8.8 \mathrm{~Hz}, 2 \mathrm{H}), 7.20(\mathrm{~d}, J=8.8 \mathrm{~Hz}, 2 \mathrm{H}), 5.42(\mathrm{~s}, 1 \mathrm{H}), 3.28-3.19(\mathrm{~m}$, 2H), $2.28(\mathrm{t}, J=7.6 \mathrm{~Hz}, 2 \mathrm{H}), 1.99-1.89(\mathrm{~m}, 2 \mathrm{H}), 1.83(\mathrm{q}, J=4.2,2.4 \mathrm{~Hz}, 2 \mathrm{H}), 1.64-1.46(\mathrm{~m}$, $4 \mathrm{H}) .{ }^{13} \mathrm{C}$ NMR $\left(100 \mathrm{MHz}, \mathrm{CDCl}_{3}\right): \delta 137.41,132.62,132.58,130.12,124.97,122.28,49.65$, 34.79, 28.10, 25.26, 22.76, 22.17. HRMS (ESI+) Calcd for $\mathrm{C}_{14} \mathrm{H}_{19} \mathrm{CIN}^{+}[\mathrm{M}+\mathrm{H}]^{+}:$236.1201; Found 236.1206.

$\mathrm{N}$-Allyl-4-chloroaniline $(\mathbf{2 3}){ }^{23}$ The general procedure was followed using $\mathrm{Ru}(\mathrm{bpy})_{3}\left(\mathrm{PF}_{6}\right)_{2}$ (0.0086 g, $0.010 \mathrm{mmol}, 1.0 \mathrm{~mol} \%), \mathrm{Cu}(\mathrm{acac})_{2}(0.0262 \mathrm{~g}, 0.100 \mathrm{mmol}, 10 \mathrm{~mol} \%), 4-$ chloroboronic acid $(0.234 \mathrm{~g}, 1.50 \mathrm{mmol}, 1.50$ equiv), allylamine $(0.075 \mathrm{~mL}, 1.00 \mathrm{mmol}, 1.00$ equiv), 2,6-lutidine (0.231 mL, $2.00 \mathrm{mmol}, 2.0$ equiv), and toluene:MeCN (1:1, $3.00 \mathrm{~mL})$. Column chromatography using 20:1 hexanes:ethyl acetate provided $\mathbf{2 3}$ as a light yellow oil (0.111 g, $0.663 \mathrm{mmol}, 66 \%) .{ }^{1} \mathrm{H}$ NMR $\left(400 \mathrm{MHz}, \mathrm{CDCl}_{3}\right): \delta 7.28(\mathrm{~d}, J=8.6 \mathrm{~Hz}, 2 \mathrm{H}), 7.06(\mathrm{~d}, J$ $=8.7 \mathrm{~Hz}, 2 \mathrm{H}), 5.89(\mathrm{td}, J=16.8,6.4 \mathrm{~Hz}, 1 \mathrm{H}), 5.82(\mathrm{~s}, 1 \mathrm{H}), 5.40-5.18(\mathrm{~m}, 2 \mathrm{H}), 3.80(\mathrm{~d}, J=6.4$ $\mathrm{Hz}, 2 \mathrm{H}) .{ }^{13} \mathrm{C}$ NMR $\left(100 \mathrm{MHz}, \mathrm{CDCl}_{3}\right): \delta 138.91,130.55,130.10,129.93,121.80,120.68,51.92$. HRMS (ESI+) Calcd for $\mathrm{C}_{9} \mathrm{H}_{11} \mathrm{CIN}^{+}[\mathrm{M}+\mathrm{H}]^{+}$: 168.0575; Found 168.0578.

1-(4-Chlorophenyl)piperidine (24). ${ }^{24}$ The general procedure was followed using $\mathrm{Ru}(\mathrm{bpy})_{3}\left(\mathrm{PF}_{6}\right)_{2}(0.0086 \mathrm{~g}, 0.010 \mathrm{mmol}, 1.0 \mathrm{~mol} \%), \mathrm{Cu}(\mathrm{acac})_{2}(0.0262 \mathrm{~g}, 0.100 \mathrm{mmol}, 10 \mathrm{~mol} \%)$, 
4-chloroboronic acid (0.234 g, $1.50 \mathrm{mmol}, 1.50$ equiv), piperidine (0.099 mL, $1.00 \mathrm{mmol}, 1.00$ equiv), 2,6-lutidine (0.231 mL, $2.00 \mathrm{mmol}, 2.0$ equiv), and toluene:MeCN (1:1, $3.00 \mathrm{~mL})$. Column chromatography using 20:1 hexanes:ethyl acetate provided $\mathbf{2 4}$ as a light yellow oil $(0.158 \mathrm{~g}, 0.805 \mathrm{mmol}, 81 \%)$ that was further purified using HPLC with a gradient of $10-60 \% \mathrm{~B}$ over $20 \mathrm{~min}$ at $5 \mathrm{~mL} / \mathrm{min}$ using a C18 $5 \mu \mathrm{m}, 9.4 \times 250 \mathrm{~mm}$ column (A: $0.1 \%$ TFA water, B: $0.1 \%$ TFA) to deliver the product as a colorless oil $(0.158 \mathrm{~g}, 0.805 \mathrm{mmol}, 81 \%) .{ }^{1} \mathrm{H} \mathrm{NMR}(400 \mathrm{MHz}$, $\left.\mathrm{CDCl}_{3}\right): \delta 9.54(\mathrm{~s}, 1 \mathrm{H}), 7.54(\mathrm{~d}, J=9.1 \mathrm{~Hz}, 2 \mathrm{H}), 7.45(\mathrm{~d}, J=9.1 \mathrm{~Hz}, 2 \mathrm{H}), 3.52-3.38(\mathrm{~m}, 4 \mathrm{H})$, $2.08(p, J=5.9 \mathrm{~Hz}, 4 \mathrm{H}), 1.73(\mathrm{p}, J=6.2 \mathrm{~Hz}, 2 \mathrm{H}) .{ }^{13} \mathrm{C} \mathrm{NMR}\left(100 \mathrm{MHz}, \mathrm{CDCl}_{3}\right): \delta$ 138.91, $130.55,130.10,129.93,121.80,120.68,51.92$. HRMS (ESI+) Calcd for $\mathrm{C}_{11} \mathrm{H}_{15} \mathrm{CIN}^{+}[\mathrm{M}+\mathrm{H}]^{+}$: 196.0888; Found 196.0893.

1-(4-Chlorophenyl)-2-methylpiperidine (25). The general procedure was followed using $\mathrm{Ru}(\mathrm{bpy})_{3}\left(\mathrm{PF}_{6}\right)_{2}(0.0086 \mathrm{~g}, 0.010 \mathrm{mmol}, 1.0 \mathrm{~mol} \%), \mathrm{Cu}(\mathrm{acac})_{2}(0.0262 \mathrm{~g}, 0.100 \mathrm{mmol}, 10 \mathrm{~mol} \%)$, 4-chloroboronic acid $(0.234 \mathrm{~g}, 1.50 \mathrm{mmol}, 1.50$ equiv), 2-methylpiperidine $(0.118 \mathrm{~mL}, 1.00$ mmol, 1.00 equiv), 2,6-lutidine ( $0.231 \mathrm{~mL}, 2.00 \mathrm{mmol}, 2.0$ equiv), and toluene:MeCN (1:1, 3.00 $\mathrm{mL}$ ). Column chromatography using 20:1 hexanes:ethyl acetate provided $\mathbf{2 5}$ as a light yellow oil $(0.140 \mathrm{~g}, 0.668 \mathrm{mmol}, 67 \%)$. IR (thin film): 3055 (w), 2916 (w), 2867 (w), 1607 (w), 1510 (w), $1244(\mathrm{~m}) .{ }^{1} \mathrm{H}$ NMR $\left(400 \mathrm{MHz}, \mathrm{CDCl}_{3}\right): \delta 7.58(\mathrm{~d}, J=8.1 \mathrm{~Hz}, 2 \mathrm{H}), 7.49(\mathrm{~d}, J=9.2 \mathrm{~Hz}, 2 \mathrm{H}), 3.72$ (d, $J=12.0 \mathrm{~Hz}, 1 \mathrm{H}$ ), 3.42 (ddq, $J=12.9,6.4,3.5 \mathrm{~Hz}, 1 \mathrm{H}$ ), $3.21(\mathrm{td}, J=12.5,3.1 \mathrm{~Hz}, 1 \mathrm{H}$ ), 2.24 (dq, $J=35.1,12.3,11.7 \mathrm{~Hz}, 2 \mathrm{H}), 1.98(\mathrm{dd}, J=33.1,14.1 \mathrm{~Hz}, 2 \mathrm{H}), 1.60(\mathrm{q}, J=14.5,12.8 \mathrm{~Hz}$, 1H), $1.17(\mathrm{~d}, J=6.5 \mathrm{~Hz}, 3 \mathrm{H}) .{ }^{13} \mathrm{C}$ NMR $\left(100 \mathrm{MHz}, \mathrm{CDCl}_{3}\right): \delta 139.38,136.24,130.86,123.53$, $63.75,58.97,31.14,23.36,22.67$, 17.96. HRMS (ESI+) Calcd for $\mathrm{C}_{12} \mathrm{H}_{17} \mathrm{CIN}^{+}[\mathrm{M}+\mathrm{H}]^{+}$: 210.1044; Found 210.1049. 
4-Chloro- $N-(($ tetrahydrofuran-2-yl)methyl)aniline (26). The general procedure was followed using $\mathrm{Ru}(\mathrm{bpy})_{3}\left(\mathrm{PF}_{6}\right)_{2}(0.0086 \mathrm{~g}, 0.010 \mathrm{mmol}, 1.0 \mathrm{~mol} \%), \mathrm{Cu}(\mathrm{acac})_{2}(0.0262 \mathrm{~g}, 0.100 \mathrm{mmol}, 10$ mol\%), 4-chloroboronic acid ( $0.234 \mathrm{~g}, 1.50 \mathrm{mmol}, 1.50$ equiv), tetrahydrofurfurylamime $(0.103$ $\mathrm{mL}, 1.00 \mathrm{mmol}, 1.00$ equiv), 2,6-lutidine (0.231 mL, $2.00 \mathrm{mmol}, 2.0$ equiv), and toluene:MeCN (1:1, $3.00 \mathrm{~mL})$. Column chromatography using $20: 1$ hexanes:ethyl acetate provided 26 as a clear oil $(0.170 \mathrm{~g}, 0.808 \mathrm{mmol}, 81 \%)$. IR (thin film): 2965 (m), $2833(\mathrm{~m}), 1623(\mathrm{w}), 1542(\mathrm{~m})$, 1107 (w), 797 (w). ${ }^{1} \mathrm{H}$ NMR (400 MHz, $\left.\mathrm{CDCl}_{3}\right): \delta 7.35$ (s, 1H), $7.24(\mathrm{~d}, J=8.9 \mathrm{~Hz}, 2 \mathrm{H}), 6.99$ (d, $J=8.9 \mathrm{~Hz}, 2 \mathrm{H}), 4.13(\mathrm{dtd}, J=8.7,6.9,3.4 \mathrm{~Hz}, 24 \mathrm{H}), 3.87(\mathrm{dt}, J=8.4,6.7 \mathrm{~Hz}, 20 \mathrm{H}), 3.74(\mathrm{dt}, J$ $=8.4,6.8 \mathrm{~Hz}, 21 \mathrm{H}), 3.28(\mathrm{dd}, J=12.4,3.4 \mathrm{~Hz}, 17 \mathrm{H}), 3.14(\mathrm{dd}, J=12.4,8.7 \mathrm{~Hz}, 16 \mathrm{H}), 2.11-$ $1.98(\mathrm{~m}, 15 \mathrm{H}), 1.97-1.84(\mathrm{~m}, 28 \mathrm{H}), 1.59(\mathrm{ddt}, J=12.1,8.1,6.9 \mathrm{~Hz}, 14 \mathrm{H}) .{ }^{13} \mathrm{C}$ NMR $(100 \mathrm{MHz}$, $\left.\mathrm{CDCl}_{3}\right): \delta 141.14,129.73,128.59,119.23,75.41,68.40,52.31,29.23,25.81 . \mathrm{HRMS}$ (ESI+) Calcd for $\mathrm{C}_{12} \mathrm{H}_{17} \mathrm{CIN}^{+}[\mathrm{M}+\mathrm{H}]^{+}$: 212.0837; Found 212.0838.

4-Chloro- $N$-(1-methoxypropan-2-yl)aniline (27). The general procedure was followed using $\mathrm{Ru}(\mathrm{bpy})_{3}\left(\mathrm{PF}_{6}\right)_{2}(0.0086 \mathrm{~g}, 0.010 \mathrm{mmol}, 1.0 \mathrm{~mol} \%), \mathrm{Cu}(\mathrm{acac})_{2}(0.0262 \mathrm{~g}, 0.100 \mathrm{mmol}, 10 \mathrm{~mol} \%)$, 4-chloroboronic acid (0.234 g, $1.50 \mathrm{mmol}, 1.50$ equiv), 1-methoxypropan-2-amine (0.105 mL, $1.00 \mathrm{mmol}, 1.00$ equiv), 2,6-lutidine $(0.231 \mathrm{~mL}, 2.00 \mathrm{mmol}, 2.0$ equiv), and toluene:MeCN (1:1, $3.00 \mathrm{~mL}$ ). Column chromatography using $20: 1$ hexanes:ethyl acetate provided $\mathbf{2 7}$ as a clear oil (0.154 g, 0.772 mmol, 77\%). IR (thin film): 3014 (m), 2848 (w), 1582 (w), 1489 (m), 1118 (w), 842 (w). ${ }^{1} \mathrm{H}$ NMR (400 MHz, $\left.\mathrm{CDCl}_{3}\right): \delta 7.29$ (d, J = 8.9 Hz, 2H), $7.11(\mathrm{~d}, J=8.9 \mathrm{~Hz}, 2 \mathrm{H}), 7.00$ (s, 1H), $3.68-3.58(\mathrm{~m}, 1 \mathrm{H}), 3.43(\mathrm{~d}, J=4.9 \mathrm{~Hz}, 2 \mathrm{H}), 3.33(\mathrm{~s}, 3 \mathrm{H}), 1.26(\mathrm{~d}, J=6.7 \mathrm{~Hz}, 3 \mathrm{H}) .{ }^{13} \mathrm{C}$ NMR $\left(100 \mathrm{MHz}, \mathrm{CDCl}_{3}\right): \delta 136.96,131.27,129.91,121.96,72.85,59.19,55.52,15.03$. HRMS (ESI+) Calcd for $\mathrm{C}_{12} \mathrm{H}_{17} \mathrm{CIN}^{+}[\mathrm{M}+\mathrm{H}]^{+}:$200.837; Found 200.0841. 
Methyl (4-chlorophenyl)-L-phenylalaninate (28). L-Phenylalanine methyl ester hydrochloride (0.216 g, $1.00 \mathrm{mmol}, 1.00$ equiv) was placed in a 1.5-dram vial with Amberlyst A-21 ion exchange resin $(0.500 \mathrm{~g})$ with $4 \mathrm{~mL}$ of $\mathrm{MeCN}$ and vigorously stirred for $10 \mathrm{~min}$. The solution was removed and placed in the reaction vial for solvent removal under reduced pressure. Following this pre-treatment of the amine coupling partner, the general procedure was followed using $\mathrm{Ru}(\mathrm{bpy})_{3}\left(\mathrm{PF}_{6}\right)_{2}(0.0086 \mathrm{~g}, 0.010 \mathrm{mmol}, 1.0 \mathrm{~mol} \%), \mathrm{Cu}(\mathrm{acac})_{2}(0.0262 \mathrm{~g}, 0.100 \mathrm{mmol}, 10$ mol\%), 4-chloroboronic acid (0.234 g, $1.50 \mathrm{mmol}, 1.50$ equiv), 2,6-lutidine (0.231 mL, 2.00 mmol, 2.0 equiv), and toluene:MeCN (1:1, $3.00 \mathrm{~mL})$. Column chromatography using 15:1 hexanes:ethyl acetate provided 28 as a clear oil $(0.198 \mathrm{~g}, 0.688 \mathrm{mmol}, 69 \%)$. IR (thin film): $3027(w), 2924(w), 1735(s), 1593(m), 1495(s), 1435(w), 1314(m), 1202(m), 813(m), 700$ (m). ${ }^{1} \mathrm{H}$ NMR $\left(400 \mathrm{MHz}, \mathrm{CDCl}_{3}\right): \delta 7.39-7.23(\mathrm{~m}, 5 \mathrm{H}), 7.14(\mathrm{~d}, J=6.5 \mathrm{~Hz}, 2 \mathrm{H}), 6.49(\mathrm{~d}, J=$

$8.8 \mathrm{~Hz}, 2 \mathrm{H}), 4.32(\mathrm{t}, J=6.2 \mathrm{~Hz}, 1 \mathrm{H}), 3.68(\mathrm{~s}, 3 \mathrm{H}), 3.13(\mathrm{dd}, J=12.4,6.2 \mathrm{~Hz}, 2 \mathrm{H}){ }^{13} \mathrm{C} \mathrm{NMR}$ $\left(100 \mathrm{MHz}, \mathrm{CDCl}_{3}\right): \delta 173.29,145.23,136.08,132.23,129.34,128.74,127.28,115.44,110.48$, 57.89, 52.34, 38.52. HRMS (ESI+) Calcd for $\mathrm{C}_{16} \mathrm{H}_{17} \mathrm{CINO}_{2}{ }^{+}[\mathrm{M}+\mathrm{H}]^{+}$: 290.0942; Found 290.0944.

\section{Supporting Information}

Copies of ${ }^{1} \mathrm{H}$ and ${ }^{13} \mathrm{C}$ NMR spectra for all numbered compounds. This material is available free of charge ACS Publications website at DOI: XXX.

\section{Author Information}

\section{Corresponding Author}

*E-mail: awommack@highpoint.edu

\section{ORCID}


Andrew J. Wommack: orcid.org/0000-0002-0889-9108

\section{Notes}

The authors declare no competing financial interest.

\section{Acknowledgements}

Research reported in this work was supported by the Summer Research Program in the Sciences (SuRPS) at High Point University, a Quest Grant from the HPU Office of Research and Sponsored Programs, and by the ACS Petroleum Research Fund (57199-UNI1). We are indebted to Drs. Greg McIntire, Erin Strickland, and many others at Ameritox for generous HRMS access. This work was performed in part at the Joint School of Nanoscience and Nanoengineering, a member of Southeastern Nanotechnology Infrastructure Corridor (SENIC) and National Nanotechnology Coordinated Infrastructure (NNCl), which is supported by the National Science Foundation (ECCS-1542174). 


\section{References}

(1) Bariwal, J.; Van der Eycken, E. C-N bond forming cross-coupling reactions: an overview Chem. Soc. Rev. 2013, 42, 9283-9303.

(2) (a) Hartwig, J. F. Approaches to Catalyst Discovery. New Carbon-Heteroatom and Carbon-Carbon Bond Formation. Pure Appl. Chem. 1999, 71, 1417-1423. (b) Muci, A.R.; Buchwald, S.L. Practical Palladium Catalysts for C-N and C-O Bond Formation. Top. Curr. Chem. 2002, 219, 131-209. (c) Fischer, C.; Koenig, B. Palladium- and Copper-Mediated N-Aryl Bond Formation Reactions for the Synthesis of Biological Active Compounds. Beilstein J. Org. Chem. 2011, 7, 59-74. (d) Fischer, C.; Koenig, B. Palladium- and Copper-Mediated N-Aryl Bond Formation Reactions for the Synthesis of Biological Active Compounds. Beilstein J. Org. Chem. 2011, 7, 59-74. (e) Forero-Cortés, P. A.; Haydl, A. M. The 25th Anniversary of the Buchwald-Hartwig Amination: Development, Applications, and Outlook. Org. Process Res. Dev. 2019 ASAP (f) Munir, I.; Zahoor, A. F.; Rasool, N.; Naqvi, S. A. R.; Zia, K. M.; Ahmad, R. Synthetic Applications and Methodology Development ofChan-Lam Coupling: A Review. Mol. Diversity 2019, 23, 215.

(3) (a) Qiao, J. X.; Lam, P. Y. S. Recent Advances in Chan-Lam Coupling Reaction: Copper-Promoted C-Heteroatom Bond Cross-Coupling Reactions with Boronic Acids and Derivatives. In Boronic Acids: Preparation and Applications in Organic Synthesis, Medicine and Materials. Hall, D. G., Ed.; Wiley-VCH: Weinheim, Germany, 2011; pp 315-361. (b) Neuville, L. Alternative and Emerging Reagents for the Arylation of Heteronucleophiles. In Copper-Mediated Cross-Coupling Reactions. Evano, G., Blanchard, N., Eds.; Wiley: Hoboken, 2013, pp 113-185. (c) Irina P. Beletskaya and Andrei V. Cheprakov . The Complementary Competitors: Palladium and Copper in C-N Cross-Coupling Reactions. Organometallics 2012, 31, 77537808.

(4) (a) Yoo, W-. J.; Tsukamoto, T.; Kobayashi, S. Visible-Light-Mediated Chan-Lam Coupling Reactions of Aryl Boronic Acids and Aniline Derivatives. Angew. Chem. Int. Ed. 2015, 54, 6587-6590. (b) Zhang, H.; Zhang, P.; Jiang, M.; Yang, H.; Fu, H. Merging Photoredox with Copper Catalysis: Decarboxylative Alkynylation of aAmino Acid Derivatives. Org. Lett. 2017, 19, 1016-1019. (c) Li, Y.; Zhou, K.; Wen, Z.; Cao, S.; Shen, X.; Lei, M.; Gong, L. Copper(II)-Catalyzed Asymmetric Photoredox Reactions: Enantioselective Alkylation of Imines Driven by Visible Light J. Am. Chem. Soc., 2018, 140, 15850-15858. (d) Levin, M. D.; Kim, S.; Toste, F. D. Photoredox Catalysis Unlocks Single-Electron Elementary Steps in Transition Metal Catalyzed Cross-Coupling. ACS Central Science 2016, 2, 293-301. (e) Skubi, K. L.; Blum, T. R.; Yoon, T. P. Dual Catalysis Strategies in Photochemical Synthesis. Chem. Rev. 2016, 116, 10035-10074.

(5) (a) Shapiro, D. A.; Renock, S.; Arrington, E.; Chiodo, L. A.; Liu, L.-X.; Sibley, D. R.; Roth, B. L.; Mailman, R. Aripiprazole, a novel atypical antipsychotic drug with a unique and robust pharmacology. Neuropsychopharmacology, 2003, 28, 1400-1411. (b) Barker, A. J.; Gibson, K. H.; Grundy, W.; Godfrey, A. A.; Barlow, J. J.; Healy, M. 
P.; Woodburn, J. R.; Ashton, S. E.; Curry, B. J.; Scarlett, L.; Henthorn, L.; Richards, L. Studies leading to the identification of ZD1839 (IRESSA): an orally active, selective epidermal growth factor receptor tyrosine kinase inhibitor targeted to the treatment of cancer. Bioorg. Med. Chem. Lett., 2001, 11, 1911-1914.

(6) (a) Collman, J. P.; Zhong, M. An Efficient Diamine Copper Complex-Catalyzed Coupling of Arylboronic Acids with Imidazoles. Org. Lett. 2000, 9, 1233-1236. (b) Collman, J. P.; Zhong, M.; Zeng, L.; Costanzo, S. The $[\mathrm{Cu}(\mathrm{OH}) \mathrm{TMEDA}]_{2} \mathrm{Cl}_{2^{-}}$ Catalyzed Coupling of Arylboronic Acids with Imidazoles in Water. J. Org. Chem. 2001, 66, 1528-1531. (c) Antilla, J. C.; Buchwald, S. L. Copper-Catalyzed Coupling of Arylboronic Acids and Amines. Organic Letters 2001, 3, 2077-2079. (d) Quach, T. D.; Batey, R. A.; Ligand- and Base-Free Copper(II)-Catalyzed C-N Bond Formation: Cross-Coupling Reactions of Organoboron Compounds with Aliphatic Amines and Anilines. Org. Lett. 2003, 5, 4397-4400. (e) Tzschucke, C. C.; Murphy, J. M.; Hartwig, J. F. Arenes to Anilines and Aryl Ethers by Sequential IridiumCatalyzed Borylation and Copper-Catalyzed Coupling. Org. Lett., 2007, 9, 761-764. (f) McGarry, K. A.; Duenas, A. A.; Clark, T. B. Selective Formation of orthoAminobenzylamines by the Copper-Catalyzed Amination of Benzylamine Boronate Esters. J. Org. Chem. 2015, 80, 7193-7204. (g) Vantourout, J. C.; Miras, H. N.; Isidro-Llobet, A.; Sproules, S.; Watson, A. J. B. Spectroscopic Studies of the Chan-Lam Amination: A Mechanism-Inspired Solution to Boronic Ester Reactivity. J. Am. Chem. Soc. 2017, 139, 4769-4779. (h) Wexler, R. P.; Nuhant, P.; Senter, T. J.; Gale-Day, Z. J. Electrochemically Enabled Chan-Lam Couplings of Aryl Boronic Acids and Anilines. Org. Lett. 2019, 21,4540-4543.

(7) Zou, Y.-Q.; Chen, J.-R.; Liu, X.-P.; Lu, L.-Q.; Davis, R. L. ; Jørgensen, K. A.; Xiao, W.-J. Highly Efficient Aerobic Oxidative Hydroxylation of Arylboronic Acids: Photoredox Catalysis Using Visible Light. Angew. Chem., Int. Ed., 2012, 124, 808812.

(8) Zuo, Z.; MacMillan, D. W. C. Decarboxylative Arylation of a-Amino Acids via Photoredox Catalysis: A One-Step Conversion of Biomass to Drug Pharmacophore. J. Am. Chem. Soc. 2014, 136, 5257-5260.

(9) (a) Evans, D. A.; Katz, J. L.; West, T. R. Synthesis of Diaryl Ethers through the Copper-Promoted Arylation of Phenols with ArylboronicAcids. An ExpedientSynthesis of Thyroxine. Tetrahedron Lett. 1998, 39, 2937-2940. (b) King, A. E.; Brunold, T. C.; Stahl, S. S. Mechanistic Study of Copper-Catalyzed Aerobic Oxidative Coupling of Arylboronic Esters and Methanol: Insights into an Organometallic Oxidase Reaction J. Am. Chem. Soc. 2009, 131, 5044-5045 (c) Casitas, A.; King, A. E.; Parella, T.; Costas, M.; Stahl, S. S.; Ribas, X. Direct observation of $\mathrm{Cu}^{\prime} / \mathrm{Cu}^{\mathrm{III}}$ redox steps relevant to Ullmann-type coupling reactions Chem. Sci., 2010, 1, 326-330.

(10)Campagna, S.; Puntoriero, F.; Nastasi, F.; Bergamini, G.; Balzani, V. Photochemistry and Photophysics of Coordination Compounds: Ruthenium,. In Photochemistry and Photophysics of Coordination Compounds I Top. Curr. Chem. Springer: Berlin, 2007, 280, 117-214. 
(11) Housecroft, C. E.; Sharpe, A. G. Inorganic Chemistry, 3rd ed.; Pearson Education: Harlow, England, 2008.

(12) Zahir, K. O.; Haim, A. Yields of singlet dioxygen produced by the reaction between the excited state of tris(bipyridine)ruthenium(II) and triplet dioxygen in various solvents. J. Photochem. Photobiol. 1992, 63, 167-172.

(13) MacNeil, S. L.; Wilson, B. J.; Snieckus, V. Anionic N-Fries Rearrangement of $\mathrm{N}$ Carbamoyl Diarylamines to Anthranilamides. Methodology and Application to Acridone and Pyranoacridone Alkaloids. Org. Lett., 2006, 8, 1133-1136.

(14) Katritzky, A. R.; Cozens, A. J. Pyrylium-mediated synthesis of unsymmetrical diarylamines from anilines. J. Chem. Soc. Perkin 1. 1983, 11, 2611-2615.

(15) Altman, R. A.; Anderson, K. W.; Buchwald, S. L. Pyrrole-2-carboxylic Acid as a Ligand for the Cu-Catalyzed Reactions of Primary Anilines with Aryl Halides. J. Org. Chem. 2008, 73, 5167-5169.

(16) Hajra, A.; Wei, Y.; Yoshikai, N. Palladium-Catalyzed Aerobic Dehydrogenative Aromatization of Cyclohexanone Imines to Arylamines. Org. Lett. 2012, 14, 54885491.

(17) Raghuvanshi, D. S.; Gupta, A. K.; Singh, K. N. Nickel-Mediated N-Arylation with Arylboronic Acids: An Avenue to Chan-Lam Coupling. Org. Lett. 2012, 14, 4326.

(18) Kampmann, S. S.; Skelton, B. W.; Wild, D. A.; Koutsantonis, G. A.; Stewart, S. G. An Air-Stable Nickel(0) Phosphite Precatalyst for Primary Alkylamine C-N CrossCoupling Reactions. Eur. J. Org. Chem. 2015, 27, 5995-6004.

(19) Zou, Q.; Wang, C.; Smith, J.; Xue, D.; Xiao, J. Alkylation of Amines with Alcohols and Amines by a Single Catalyst under Mild Conditions. Chem. Eur. J. 2015, 21, 9656-9661.

(20) Huang, M.; Hou, J.; Yang, R.; Zhang, L.; Zhu, X.; Wan, Y. A catalyst system, copper/N-methoxy-1H-pyrrole-2-carboxamide, for the Synthesis of phenothiazines in poly(ethylene glycol). Synthesis, 2014, 46, 3356-3364.

(21) Sreedhar, B.; Reddy, P. S.; Devi, D. K. Direct One-Pot Reductive Amination of Aldehydes with Nitroarenes in a Domino Fashion: Catalysis by Gum-AcaciaStabilized Palladium Nanoparticles. J. Org. Chem. 2009, 74, 8806-8809.

(22) Zang, Z. -L.; Karnakanti, S.; Zhao, S.; Hu, P.; Wang, Z.; Shao, P. -L.; He, Y. Synthesis of Spiro-dihydroquinoline and Octahydrophenanthrene Derivatives via Palladium-Catalyzed Intramolecular Oxidative Arylation. Org. Lett. 2017, 19, 13541357.

(23) Tao, Y.; Wang, B.; Wang, B.; Qu, L.; Qu, J. Highly Efficient and Regioselective Allylation with Allylic Alcohols Catalyzed by $\left[\mathrm{Mo}_{3} \mathrm{~S}_{4} \mathrm{Pd}\left(\eta^{3}\right.\right.$-allyl)] Clusters. Org. Lett. 2010, 12, 2726-2729.

(24) Vantourout, J. C.; Law, R. P.; Isidro-Llobet, A.; Atkinson, S. J.; Watson, Allan J. B. Chan-Evans-Lam Amination of Boronic Acid Pinacol (BPin) Esters: Overcoming the Aryl Amine Problem J. Org. Chem. 2016, 81, 3942-3950. 